\title{
A Scaling Law for Computational Imaging Using Spherical Optics
}

\author{
Oliver S. Cossairt ${ }^{1, *}$, Daniel Miau ${ }^{1}$, and Shree K. Nayar ${ }^{1}$ \\ 1 Computer Science Department, Columbia University, \\ 400 West 120th St., New York, NY 10027, USA \\ *Email: ollie@cs.columbia.edu
}

The resolution of a camera system determines the fidelity of visual features in captured images. Higher resolution implies greater fidelity, and thus greater accuracy when performing automated vision tasks such as object detection, recognition, and tracking. However, the resolution of any camera is fundamentally limited by geometric aberrations. In the past, it has generally been accepted that the resolution of lenses with geometric aberrations cannot be increased beyond a certain threshold. We derive an analytic scaling law showing that, for lenses with spherical aberrations, resolution can be increased beyond the aberration limit by applying a post-capture deblurring step. We then show that resolution can be further increased when image priors are introduced.

Based on our analysis, we advocate for computational camera designs consisting of a spherical lens shared by several small planar sensors. We show example images captured with a proof-of-concept gigapixel camera, demonstrating that high resolution can be achieved with a compact form factor and low complexity. We conclude with an analysis on the trade-off between performance and complexity for computational imaging systems with spherical lenses.

(C) 2011 Optical Society of America

OCIS codes: 080.1010, 080.3620, 100.1830, 100.2000, 110.1758

\section{Introduction}

High resolution cameras enable images to be captured with significantly more details than the human eye can detect, revealing information that was completely imperceptible to the

photographer at the time of capture. These cameras allow humans to explore minute details 
of a scene that may have otherwise been overlooked (see Figure 2), benefitting a variety of applications including surveillance, inspection, and forensics. Because the performance of low-level automated vision tasks depend highly on the amount image detail available, greater resolution also helps with computer vision tasks such as object detection, recognition and tracking. For these reasons and more, there is increasing demand for cameras with even higher resolution than what is commercial available today. At present, highly specialized gigapixel imaging systems are being developed for aerial surveillance [1].

While CMOS and CCD technologies have improved to the point that imaging sensors with pixels in the $1 \mu \mathrm{m}$ range have been demonstrated [2], it remains a huge challenge to design and manufacture lenses which have the resolving power to match the resolution of such a sensor. This is because the number of resolvable points for a lens, referred to as the SpaceBandwidth Product (SBP) [3], is fundamentally limited by geometrical aberrations. Ideally, all lenses would be diffraction limited so that increasing the scale of a lens while keeping FOV fixed would increase SBP. Unfortunately, SBP reaches a limit due to geometrical aberrations.

There are two common approaches that are taken to increase SBP in the face of this fundamental limit. The first is to just accept the loss in resolution and increase sensor size. As an example, consider the commercially available F/8 500mm focal length Schneider ApoSymmar lens. If this lens were diffraction limited, it would be capable of resolving a gigapixel image on a 5" x5" sensor. However, because of geometrical aberrations, a sensor size of nearly

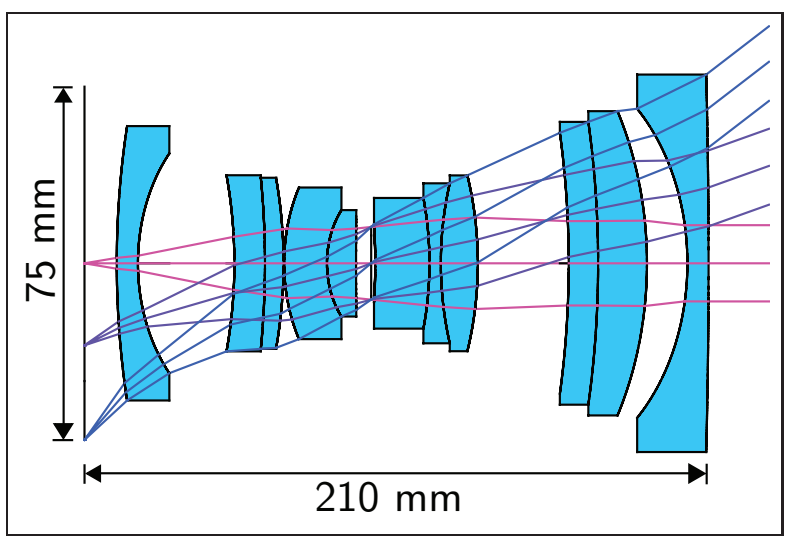

(a) An F/4 75mm focal length lens

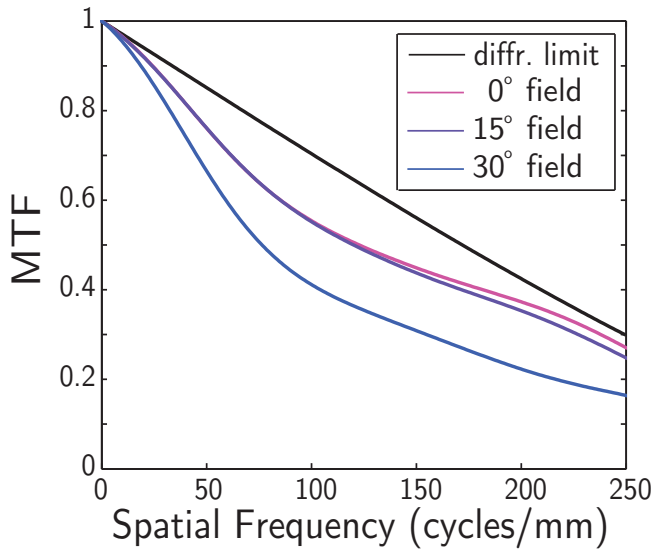

(b) The MTF of the lens in (a)

Fig. 1. (a) An F/4 75mm lens design capable of imaging one gigapixel onto a $75 \times 75 \mathrm{~mm}$ sensor. This lens requires 11 elements to maintain diffraction limited performance over a $60^{\circ} \mathrm{FOV}$. (b) The MTF at different field positions on the sensor. 
$12 " \mathrm{x} 12 "$ is necessary to resolve a full gigapixel image.

The second approach taken to increase SBP is to increase complexity as a lens is scaled up. Introducing more optical surfaces increases the degrees of freedom in lens optimization, which can be used to reduce geometric aberrations and achieve diffraction limited performance. Consider the F/4 75mm focal length lens shown in Figure 1. The lens is diffraction limited over a $60^{\circ} \mathrm{FOV}$ so that a gigapixel image can be resolved on a $75 \mathrm{mmx} 75 \mathrm{~mm}$ surface, much smaller than for the Apo-Symmar. The increase in performance comes at a great cost, however. The design consists of 11 different elements, ranging from 60-100mm in diameter, resulting in a lens that is both expensive to produce and difficult to align.

The following are the main contributions of our paper.

A Scaling Law for Computational Imaging. We present a new approach to increase SBP - the use of computations to correct for geometrical aberrations. In conventional lens design, resolution is limited by the spot size of the lens. For a lens with aberrations, spot size increases linearly with the scale of the lens. For a computational imaging system, resolution is related to deblurring error. We observe, however, that for a lens with spherical aberrations, deblurring error does not increase linearly with lens scale. We use this remarkable fact to derive a scaling law that shows that computational imaging can be used to develop cameras with very high resolution while maintaining low complexity and small size. First, we analytically derive a closed form expression for the Point spread Function (PSF) and Optical Transfer Function (OTF) of a lens with spherical aberration. We then use this expression to derive a closed form solution for the deblurring error as a function of lens scale. We go on to show how deblurring performance improves when image priors are introduced.

Gigapixel Computational Camera Designs. We present an imaging architecture that consists of a large ball lens shared by an array of small planar sensors coupled with a deblurring step. Due to our monocentric optical design, field-dependent aberrations are suppressed, and the primary aberrations are spherical and axial chromatic, which are known to code images in a manner that is invertible via post-processing [4] [5] [6] [7]. We demonstrate a proof-of-concept gigapixel camera that is implemented by sequentially scanning a single sensor to emulate an array of tiled sensors. In addition, we present a single element gigapixel camera design with a contiguous FOV.

Performancs vs. Complexity Analysis. We advocate the use of deblurring to remove the effects of aberrations. However the quality of deblurred images depends on the MTF of the lens, and a diffraction limited lens always has the best possible performance. Unfortunately, achieving diffraction limited performance often requires increasing the complexity of the lens, usually by increasing the number of surfaces. Lenses with greater complexity are typically larger, heavier, more expensive to manufacture, and more difficult to align. We analyze the trade-off between performance and complexity for the special case of spherical optics. 


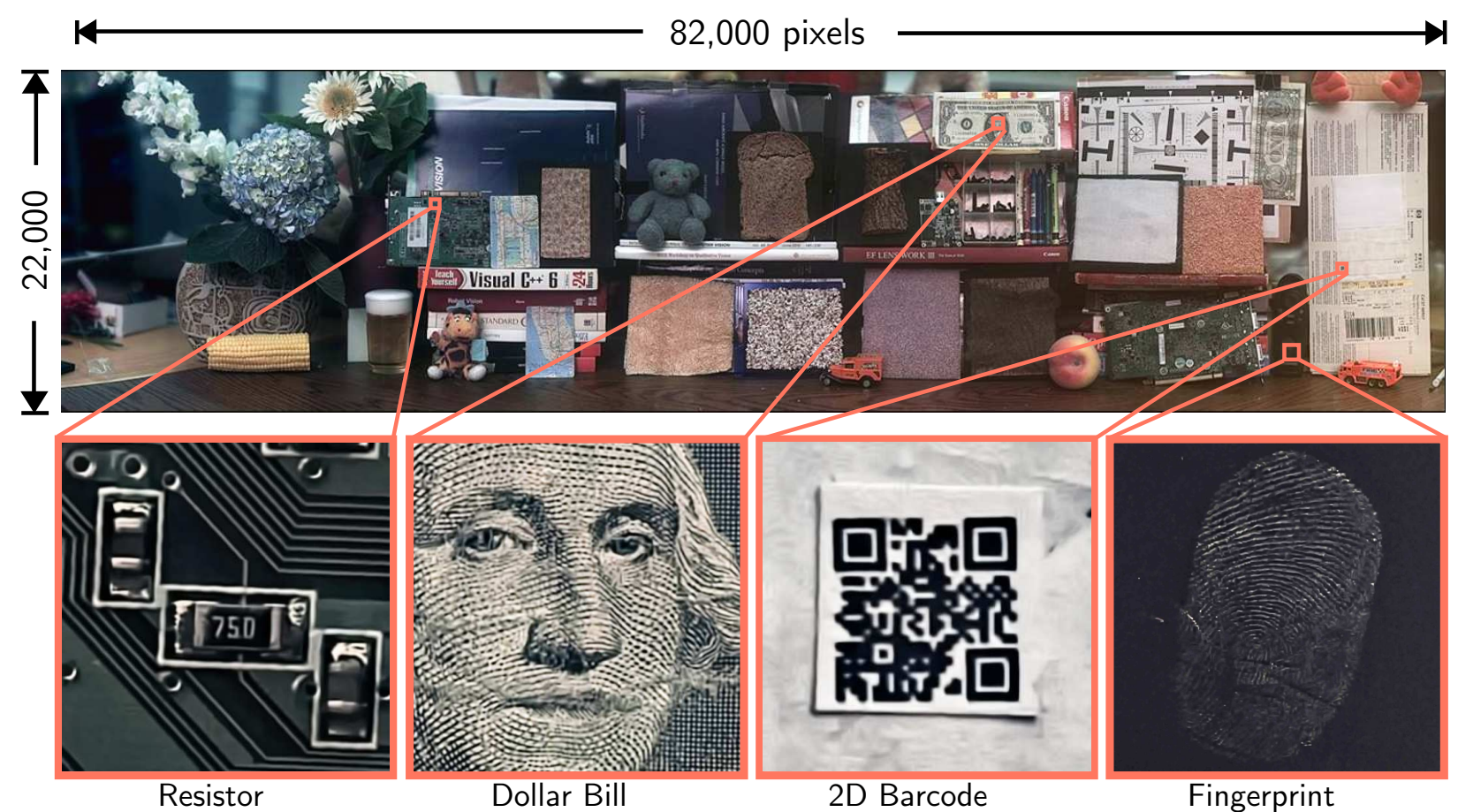

Fig. 2. A $\mathbf{1 . 7}$ gigapixel image captured using the implementation shown in Figure 13. The image dimensions are 82,000 x 22,000 pixels, and the scene occupies a $126^{\circ} \times 32^{\circ}$ FOV. From left to right, insets reveal the label of a resistor on a PCB board, the stippling print pattern on a dollar bill, a miniature 2D barcode pattern, and the fine ridges of a fingerprint on a remote control. The insets are generated by applying a $60 \mathrm{x}-200 \mathrm{x}$ digital zoom to the above gigapixel image. Please visit http://gigapan.org/gigapans/0dca576c3a040561b4371cf1d92c93fe/ to view this example in more detail.

\section{Related Work}

\section{A. Large Format Imaging Systems}

A few custom high resolution imaging systems have been developed using large format lenses. These include systems built with commercial lenses that sequentially scan a large image plane surface [8] [9], as well as a system with a custom lens that is photographed on film and later converted to a digital image [10]. These are special purpose cameras that are extremely large (FL > 500mm). In Section 8 we show that it is possible to capture images at comparable resolutions with a much smaller form factor. 


\section{B. Camera Arrays and Multiscale Optics}

Camera arrays have been used to capture high resolution images by tiling multiple sensors paired with a complex lens [11] [12]. However, a camera array for gigapixel imaging would be prohibitively large and expensive because it would require tiling an array of long focal length lenses. A related approach taken by Brady and Hagen [13] is to use a multiscale optical system consisting of a large single element lens coupled with an array of smaller optical elements, each unique and coupled with a different sensor. The advantage of this approach is that it is a compact design that can correct for geometrical aberrations. The disadvantage is that the system requires a large number of different optical elements, which may be difficult to manufacture and align.

\section{C. Monocentric Optics and Curved Sensors}

Monocentric optical designs are free of field dependent aberrations because they are completely symmetric: the image plane and each lens surface lay on concentric spheres. Monocentric designs date back to the Sutton Panoramic Lens (1859), and later the Baker Ball Lens (1942) [14]. Luneburg proposed the use of a monocentric lens with varying index of refraction to correct for aberrations [15]. Rim et. al proposed a small diffraction limited camera consisting of a ball lens and curved sensor [16]. Krishnan and Nayar proposed the use of a large ball lens and spherical sensor together with deblurring to create a single viewpoint, fully spherical FOV camera [17]. While several researchers have made progress towards developing curved sensors [18] [19] [20], the technology is not yet ready for commercialization.

Recently, Marks and Brady proposed a 7-element large format monocentric lens called the Gigagon [21], which the authors suggest using with a large array of planar sensors. To our knowledge this system has yet to be implemented, but is similar in architecture to some of the designs we propose ${ }^{1}$. Our approach is fundamentally different in that we show how computations can be used to achieve the desired resolution while reducing complexity.

\section{D. Computational Imaging}

In the 90's, Cathey and Dowski proposed a hybrid optical-signal processing system which uses a cubic phase plate to extend depth of field [22]. Later they showed that the same element can be used to reduce the complexity of infrared cameras [23]. Robinson and Stork observed that spherical aberrations are easily invertible via image processing, and proposed the use of simpler lens designs based on this principle [4] [5] [24]. Guichard et. al [6] and Cossairt and Nayar [7] observed that the effects of axial chromatic aberrations can be inverted using a method that is inexact, but produces images that look good.

\footnotetext{
${ }^{1}$ Similar camera designs are also being pursued by the DARPA MOSAIC project, led by David J. Brady. Terrapixel Imaging, ICCP '10 Invited Talk, Mar 2010.
} 


\section{Diffraction Limited Resolution}

Lohmann originally observed that lenses obey certain scaling laws that determine how resolution increases as a function of lens size [25]. Consider a lens with focal length $f$, aperture diameter $D$, and image size $\Delta x$ by $\Delta y$. We introduce a scaling factor $M$, which is defined such that $M=1$ corresponds to a focal length of $f=1 \mathrm{~mm}$. If we scale the lens by a factor of $M$, then $f, D, \Delta x$ by $\Delta y$ are all scaled by $M$, but the $\mathrm{F} / \#$ and FOV of the lens remain unchanged. If, when we scale the lens, the minimum resolvable spot size has not also increased by a factor of $M$, then we have increased the total number of points that can be resolved. The number of resolvable points for a lens is referred to as the Space-Bandwidth Product (SBP) [3]. SBP is a unit-less quantity that tells us the number of distinct points which can be measured over a given FOV.

The minimum spot diameter of a lens due to diffraction is $\delta_{d} \approx \lambda F / \#$, where $\lambda$ is the wavelength of light. Since this quantity is independent of lens scale, the SBP for a diffractionlimited lens is

$$
R_{\text {diff }}(M)=\frac{M^{2} \Delta x \Delta y}{(\lambda F / \#)^{2}} .
$$

The SBP increases quadratically with the scaling factor $M$ (see the red curve in Figure 3).

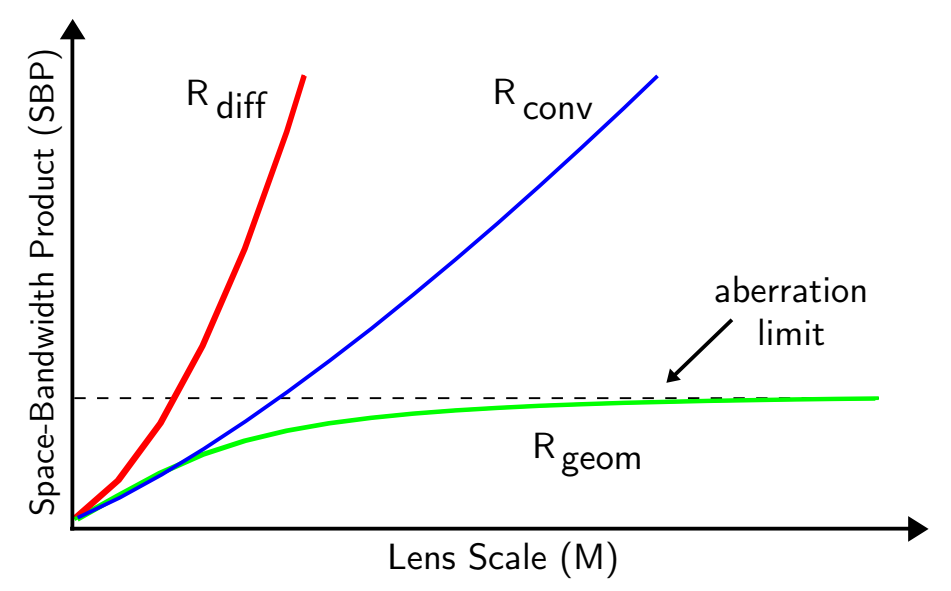

Fig. 3. A plot showing how Space-Bandwidth Product (SBP) increases as a function of lens size for a perfectly diffraction limited lens $\left(R_{\text {diff }}\right)$, a lens with geometric aberrations $\left(R_{\text {geom }}\right)$, and a conventional lens design whose $\mathrm{F} / \#$ increases with lens size $\left(R_{\text {conv }}\right)$. 


\section{Aberrations and Image Quality}

Ideally, all lenses would be diffraction limited, and resolution would scale quadratically with lens size. Unfortunately, the resolution of most lenses is limited not by diffraction, but by geometrical aberrations. This is because there is no lens shape that can produce a perfect focus for all points on the image plane. The best we can do is to reduce aberrations to the point that their effect is small compared to diffraction.

\section{A. Aberration Theory}

The Optical Path Difference (OPD) generalizes the concept of lens aberrations. The OPD measures the distance between an ideal focusing wavefront and the actual wavefront propagating through the lens as a function of normalized coordinates in the pupil plane (see Figure 4). For radially symmetric lenses, the generalized OPD is a function of 2-D polar coordinates $\{\rho \in[-1,1], \phi \in[0, \pi]\}$ in the aperture plane, and the radial coordinate $r$ on the sensor plane. In optical design, the OPD $W(\rho, \phi, r)$ is typically expressed as a Siedel polynomial, where each term in the polynomial represents a different type of aberration:

$$
W(\rho, \phi, r)=\sum_{i, j, k} W_{i j k} r^{i} \rho^{j} \cos ^{k} \phi .
$$

For instance, $W_{020}, W_{040}, W_{131}$ represent the amounts of defocus, spherical aberration, and coma, respectively. For spherical optical systems, the aberrations become independent of

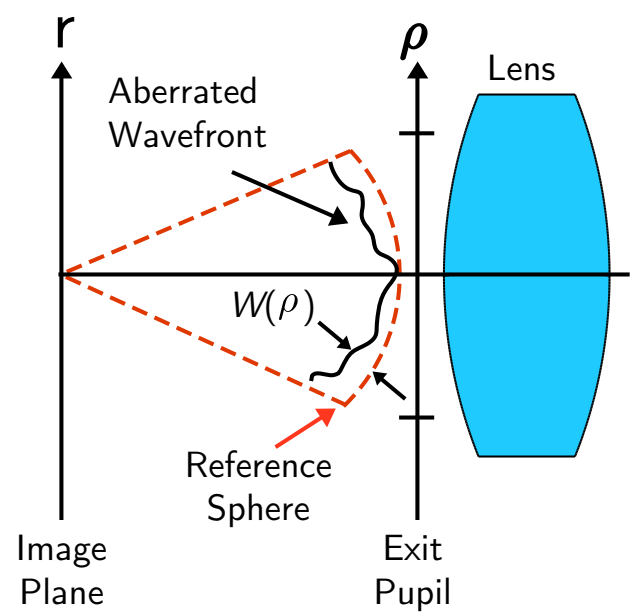

Fig. 4. The OPD $W(\rho)$ of a lens is the path difference between an ideal spherical wavefront and the aberrated wavefront propagating from the exit pupil of the lens. 
position on the sensor due to the symmetry of the system. In this case, the OPD becomes

$$
W(\rho)=\sum_{i, j, k} W_{i j k} \rho^{j},
$$

in which case defocus and spherical aberration become the dominant aberrations. For a thin lens, the spherical aberration coefficient $W_{040}$ can be shown to be [26]

$$
W_{040}=\frac{\sigma_{I}}{512} \frac{D}{F / \#^{3}},
$$

where $D$ is again the diameter of the lens aperture, and $\sigma_{I}$ is the structural coefficient (a constant that depends only on index of refraction and is usually in the range $\sigma_{I}=5-15$ ).

\section{B. The Aberration Induced PSF}

When a lens exhibits aberrations, it can no longer produce a perfect focus. A perfectly focusing lens produces a Point Spread Function (PSF) that is a delta function, which produces the sharpest focus possible. Diffraction and geometric aberrations cause the PSF to deviate from this ideal shape. The OPD can be used to calculate the PSF produced by an optical system with aberrations. If the aberrations are relatively small, then the effect of diffraction

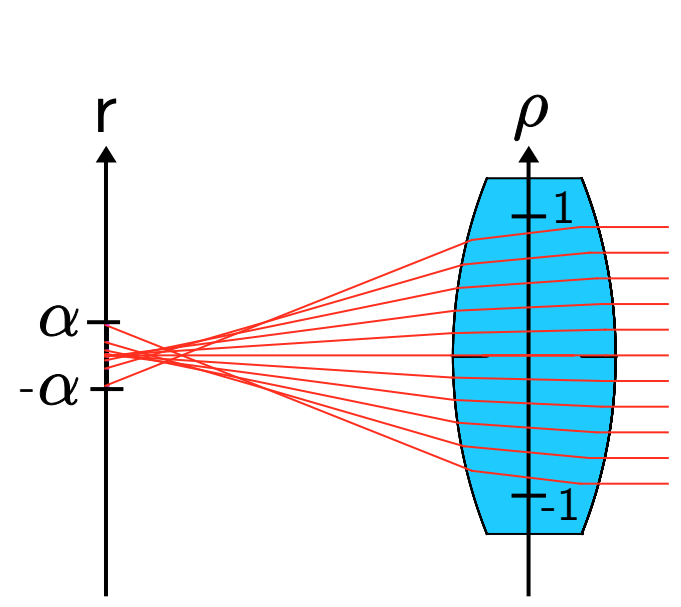

(a) A singlet with aberrations

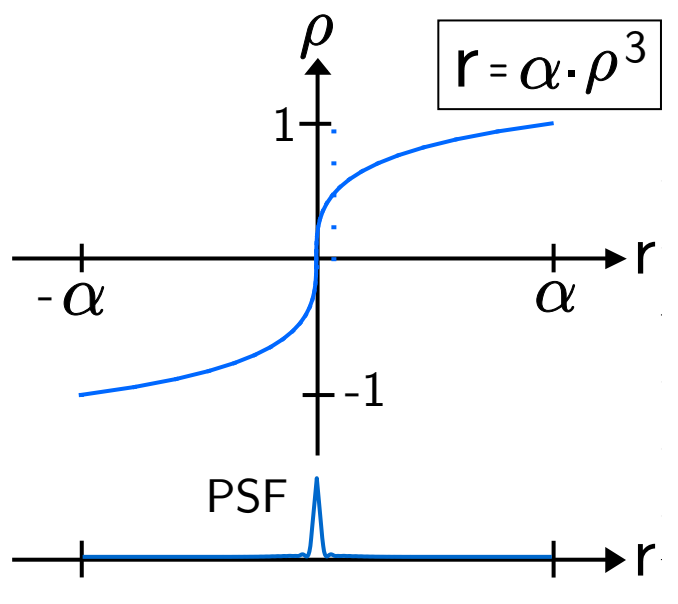

(b) The rayfan and PSF of (a)

Fig. 5. (a) A singlet lens with strong spherical aberrations. (b) The rayfan shows ray position on the sensor plane as a function of position in the lens aperture. The PSF has a strong peak because rays are concentrated around the center of the image plane. The PSF is support is enclosed in an area of radius $\alpha$. 
needs to be considered and Fourier Optics must be used to derive the correct PSF shape. If the aberrations are large, however, the PSF can be derived using geometric optics. Since rays propagate perpendicular to the aberrated wavefront, we can use the OPD to determine where each ray pierces the sensor plane. The transverse ray-aberration curve $r=T(\rho)$ gives the position of a ray in the sensor coordinates $r$ as a function of coordinates in the pupil plane $\rho$. For a point source at infinity, this is given by [26]:

$$
T(\rho)=2 F / \# \frac{d W}{d \rho} .
$$

For a lens with spherical aberrations, the transverse aberration curve is given by (see Figure 5(b))

$$
\begin{aligned}
T(\rho) & =\frac{\sigma_{I}}{64} \frac{D}{F / \#^{2}} \rho^{3} \\
& =\alpha \rho^{3},
\end{aligned}
$$

where $\alpha$ is the spherical aberration coefficient (usually called $S A 3$ ). Because $\rho$ is given in normalized coordinates, the full support of the PSF falls within a circle of radius $\alpha$ (see Figure 5(b)). From Equation 7 it is clear that if we scale the lens uniformly by a factor of $M$ (such that the $\mathrm{F} / \#$ remains constant), $\alpha$ increases by the same factor.

We can think of the ray-aberration curve as an integration curve in a radially symmetric light field phase space [27] [28] [29]. That is, we can write the light field of a point source propagating through an aberrated lens as

$$
L(r, \rho)=\frac{1}{\pi} \sqcap(\rho) \frac{\delta(r-T(\rho))}{\pi|r|},
$$

where

$$
\Pi(\rho)= \begin{cases}1 & \text { if }|\rho|<1 \\ 0 & \text { otherwise }\end{cases}
$$

is the tophat function. The advantage of the light field representation is that the PSF can be found by integrating over the aperture coordinates. We consider the general monomial OPD $W(\rho)=\alpha /(n+1) \rho^{n+1}$ which leads to the ray-aberration curve $T(\rho)=\alpha \rho^{n}$. We note that taking the modulus of the radial coordinate inside the ray aberration curve so that $T(\rho)=\alpha|\rho|^{n}$ does not alter the PSF. The Point Spread Function (PSF) of the lens can then be written as (for a derivation see Appendix A) 


$$
\begin{aligned}
P(r) & =\pi \int_{-\infty}^{\infty} L(r, \rho)|\rho| d \rho \\
& =\frac{1}{\pi n \alpha^{2 / n}} \sqcap\left(\frac{r}{\alpha}\right)|r|^{2 / n-2} .
\end{aligned}
$$

The PSF can be shown to be unit normalized so that the integral of the PSF over sensor coordinates is equal to 1 (see Appendix B). The PSF for a lens with spherical aberrations is then written as

$$
P(r)=\frac{3}{2 \pi \alpha^{2 / 3}} \sqcap\left(\frac{r}{\alpha}\right)|r|^{-4 / 3} .
$$

\section{Aberrations and Resolution Scaling Laws}

\section{A. The Classical Aberration Limit to Resolution}

For a diffraction limited lens, the SBP increases quadratically with the scaling factor $M$. However, the SBP of a lens also depends on the diameter of the blur circle caused by geometric aberrations. We introduce the variable $\delta_{g}$, which represents the geometric spot size at lens scale $M=1$, which we recall corresponds to a focal length of $f=1 \mathrm{~mm}$. Lohmann argues that the combined blur area when diffraction and aberration are taken into account can be

expressed as the sum $\delta_{d}^{2}+\delta_{g}^{2}$. Since geometric blur increases linearly with the scaling factor $M$, the SBP becomes [25]

$$
R_{\text {geom }}(M)=\frac{M^{2} \Delta x \Delta y}{(\lambda F / \#)^{2}+M^{2} \delta_{g}^{2}} .
$$

In this case, the SBP plateaus at $\Delta x \Delta y / \delta_{g}^{2}$ when the lens is no longer diffraction limited and $M \delta_{g}>\lambda F / \#$ (see the green curve in Figure 3). For this reason, lens designers typically seek to balance lens aberrations in an effort to minimize the blur circle. For example, defocus can be introduced into a lens with spherical aberrations in order to reduce the geometric blur circle. From a classical perspective, this strategy increases resolution because it decreases the spot size of the lens. As we will show in Section 6 however, this strategy is not desirable from a computational imaging perspective because it reduces the conditioning of the PSF, introducing more deblurring error.

\section{B. The Scaling Law for Conventional Lens Design}

The geometric blur size can always be decreased by stopping down a lens. As a result lens designers typically increase the $\mathrm{F} / \#$ as a lens is scaled up. A general rule of thumb is that the $\mathrm{F} / \#$ is increased such that the focal length in $m m$ is approximately equal to $(\mathrm{F} / \#)^{3}$. Many commercially available lenses follow this general trend (see Figure 6). For instance, the 
$500 \mathrm{~mm}$ focal length Schneider Apo-Symmar operates at $\mathrm{F} / 8$, and $8^{3} \approx 500$. This heuristic $\mathrm{F} / \#$ scaling law has a special significance for lenses with spherical aberration. Then the geometric blur size $\delta_{g}$ is proportional to the spherical aberration coefficient $\alpha$, and from Equation 7

$$
\alpha=\frac{\sigma_{I}}{64} \frac{D}{F / \#^{2}}=\frac{\sigma_{I}}{64} \frac{f}{F / \#^{3}} .
$$

Thus, if the $\mathrm{F} / \#$ increases with the cube root of the focal length, the geometric blur size $\delta_{g}$ becomes independent of the scaling factor $M$. However, the diffraction blur size now increases as a function of scale so that $\delta_{d}=\lambda M^{1 / 3}$. Then (see the blue curve in Figure 3 ) the SBP becomes [25]

$$
R_{\text {conv }}(M)=\frac{M^{2} \Delta x \Delta y}{\lambda^{2} M^{2 / 3}+\delta_{g}^{2}} .
$$

Equation 15, derived by Lohmann, is a scaling law that tells us generally how SBP increases with lens size for a conventional lens design. The equation says that when $M$ is large, the diffraction spot size dominates geometric blur. In this regime, the scaling follows the behavior:

$$
R_{\text {conv }}(M) \propto M^{4 / 3}
$$

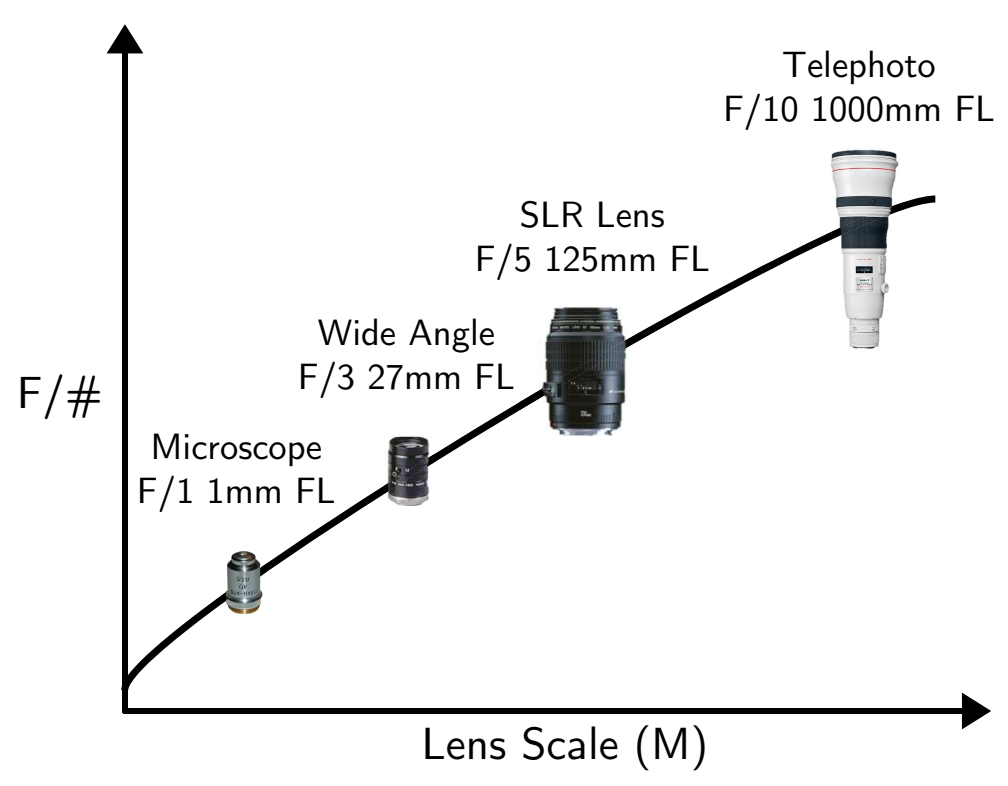

Fig. 6. For conventional lens designs, the F/\# typically scales with the cube root of the focal length in millimeters. 
which overcomes the resolution threshold set by the aberration limit, but does not attain the ideal $M^{2}$ behavior of the diffraction limited scaling law.

\section{Computational Imaging}

Conventional optical systems are based on the centuries old tradition of modeling optical systems as isomorphic mappings between scene radiance and pixel intensity. In a conventional camera, it is assumed that the brightness measured at a single pixel corresponds directly to the radiance of a single scene point. In the computational imaging paradigm, we consider the optical system as a channel that linearly encodes an image according to the image formation equation

$$
\mathbf{y}=A \mathbf{x}+\nu,
$$

where $\mathbf{y} \in \mathrm{R}^{M}$ is a vector consisting of a lexicographic re-ordering of the $M$ measured pixel intensities, $A$ is an $M \mathrm{x} N$ matrix, $\mathrm{x} \in \mathrm{R}^{N}$ is a vector of $N$ unknown discreet scene radiance values, and $\nu \in \mathrm{R}^{M}$ is a vector representing the noise measured at each pixel, typically assumed to be gaussian so that $\nu \sim \mathcal{N}\left(0, \sigma_{n}^{2} I\right)$. The vector of unknown radiance values $\mathbf{x}$ is a discretization of the continuous radiance distribution representing a latent focused image, and in most cases the system is fully determined so that $M=N$. The most important distinction between conventional imaging and computational imaging is that the former maps each unknown radiance value to a single pixel measurement, while the latter maps a linear combination of unknowns to each pixel measurement.

In the analysis that follows, we assume the optical system is shift invariant, in which case the observation can be modeled as a convolution between the lens PSF and the unknown scene radiance. Convolution can be expressed compactly in the Fourier domain as the product between the Fourier transform of the PSF, referred to as the Optical Transfer Function (OTF), and the Fourier transform of the scene radiance. In our discreet framework, we denote the PSF by the vector $\mathbf{p}$ and the OTF by the vector $\hat{\mathbf{p}}=\mathrm{F} \mathbf{p}$, where $\mathrm{F}$ is the Fourier matrix. The matrix $A$ becomes a cyclic matrix such that $A_{i, j-i}=p_{i}$ with the special property that it can be written as $A=\mathrm{F} \Lambda \mathrm{F}$, where $\Lambda$ is a diagonal matrix and $\Lambda_{i i}=\hat{p}_{i}$. Then the

image formation equation can be written as a sparse set of linear equations in the Fourier domain:

$$
\hat{\mathbf{y}}=\Lambda \hat{\mathbf{x}}+\hat{\nu}
$$

where the ^ operator denotes multiplication with the Fourier matrix F. 


\section{A. Image Deblurring}

In the conventional imaging paradigm, pixel measurements correspond directly to scene radiance values. In the computational imaging paradigm, the unknown image $\mathbf{x}$ is blurred by the matrix $A$. To deblur the captured image $\mathbf{y}$ we must invert Equation 17. If the PSF is well conditioned, then the OTF contains no zero crossings and the matrix $A$ is full rank and invertible, and we can estimate the unknown radiance $\mathbf{x}^{*}$ as

$$
\hat{\mathbf{x}}^{*}=\Lambda^{-1} \hat{\mathbf{y}} .
$$

Equation 19 is a sparse set of linear equations such that the estimate $\mathbf{x}^{*}$ is found simply by taking the ratio of Fourier coefficients

$$
\hat{x}_{i}^{*}=\hat{y}_{i} / \hat{p}_{i} .
$$

The final estimate can then be found by simply taking an inverse Fourier Transform. Unfortunately, we cannot recover the unknown image exactly because the original measurements were corrupted by noise. In order to quantify the quality of the deblurred image, we use the mean squared deblurring error $\sigma_{d}^{2}$ as a metric, which is defined as the expected mean squared difference between the deblurred image $\mathbf{x}^{*}$ and the ground truth image $\mathbf{x} . \sigma_{d}^{2}$ measures the variance of noise artifacts induced by the deblurring process. In our shift invariant system, this can be written as

$$
\begin{aligned}
\sigma_{d}^{2} & =\frac{1}{N} E\left[\left\|\mathbf{x}^{*}-\mathbf{x}\right\|^{2}\right] \\
& =\frac{\sigma_{n}^{2}}{N} \sum_{i=1}^{N} \frac{1}{\left\|\hat{p}_{i}\right\|^{2}},
\end{aligned}
$$

where $E$ denotes taking the expectation with respect to the noise $\nu$. Equation 22 says that, when naive deblurring is applied, the deblurring error is a product between the noise variance and the average squared reciprocal of the OTF.

\section{B. Spherical Aberrations and Deblurring}

In Section 4.B showed that the spherical aberration coefficient $\alpha$ scales linearly with lens size, and we derived the analytic expression for the PSF of a lens with spherical aberrations, given by Equation 12. From this expression, we can derive the OTF of the lens. For a radially symmetric PSF $p(r)$, the OTF $\hat{p}(q)$ can be found by applying the zero order Hankel transform:

$$
\hat{p}(q)=2 \pi \int_{0}^{\infty} J_{0}(q r) p(r) r d r
$$


where $J_{0}(r)$ is the Bessel function of the first kind. For the PSF given by Equation 12, the OTF becomes

$$
\begin{aligned}
\hat{p}(q) & =\frac{2}{\alpha^{2 / 3}} \int_{0}^{\alpha} J_{0}(q r) r^{-1 / 3} d r \\
& ={ }_{1} F_{2}\left(\left\{\frac{1}{3}\right\},\left\{1, \frac{4}{3}\right\},-\frac{\alpha^{2} q^{2}}{4}\right),
\end{aligned}
$$

where ${ }_{1} F_{2}(a ; b, c ; d)$ is the Generalized Hypergeometric Function [30]. Figure 7 shows a comparison between the OTF calculated analytically using Equation 24 and the OTF calculated numerically using the Geometric MTF feature in Zemax Optical Design Software [31]. The OTF is calculated at a variety of lens scales corresponding to spherical aberration coefficients $\alpha=\{5 \mu m, 13 \mu m, 100 \mu m\}$, and the results are highly consistent in all cases.

With an equation for the OTF, it is possible to derive an analytic expression for the deblurring error. In the continuous domain, the deblurring error from Equation 22 becomes

$$
\sigma_{d}^{2}=\frac{2 \sigma_{n}^{2}}{\Omega^{2}} \int_{0}^{\Omega} \frac{1}{\|\hat{p}\|^{2}(q)} q d q,
$$

where the signal is assumed to be bandlimited by the nyquist frequency $\Omega$. Unfortunately,

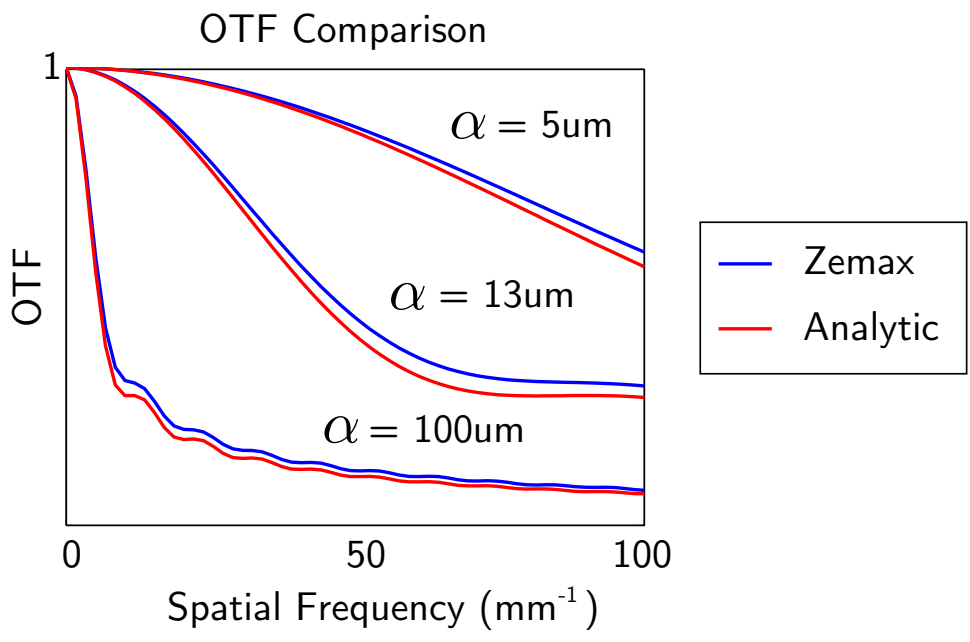

Fig. 7. A comparison of the OTF for a lens with spherical aberration calculated using Zemax (the blue curves) and using our analytic formula (red curves). The OTF is calculated at various lens scales corresponding to spherical aberration coefficients of $\alpha=\{5 \mu m, 13 \mu m, 100 \mu m\}$ 
there is no closed form solution for the expression in Equation 26 after substituting the Hypergeometric function, so we instead approximate the OTF using the following equation:

$$
\begin{aligned}
\hat{p}(q) & =\frac{2}{\alpha^{2 / 3}} \int_{0}^{\infty} J_{0}(q r) r^{-1 / 3} d r \\
& =\frac{2 \Gamma(7 / 6)}{\sqrt{\pi} \alpha^{2 / 3}},
\end{aligned}
$$

where $\Gamma$ is the gamma function. Equation 27 essentially approximates the PSF as having infinite support, which is accurate for large amounts of spherical aberration, but decreases in accuracy as the spherical aberration approaches zero. Figure 8 shows a comparison of the OTF calculated using using our analytic formula (red curves) and using the approximation for the OTF given by Equation 27. The OTF is calculated at various lens scales corresponding to spherical aberration coefficients of $\alpha=\{20 \mu m, 50 \mu m, 200 \mu m\}$. As the amount of spherical aberrations increase, the approximation increases in accuracy.

Substituting the approximate MTF from Equation 27 into the expression in Equation 26 gives us an analytic expression for the deblurring error:

$$
\sigma_{d}=\sigma_{n} \sqrt{\frac{3 \pi}{2}} \frac{(\Omega \alpha)^{2 / 3}}{2 \Gamma(7 / 6)} .
$$

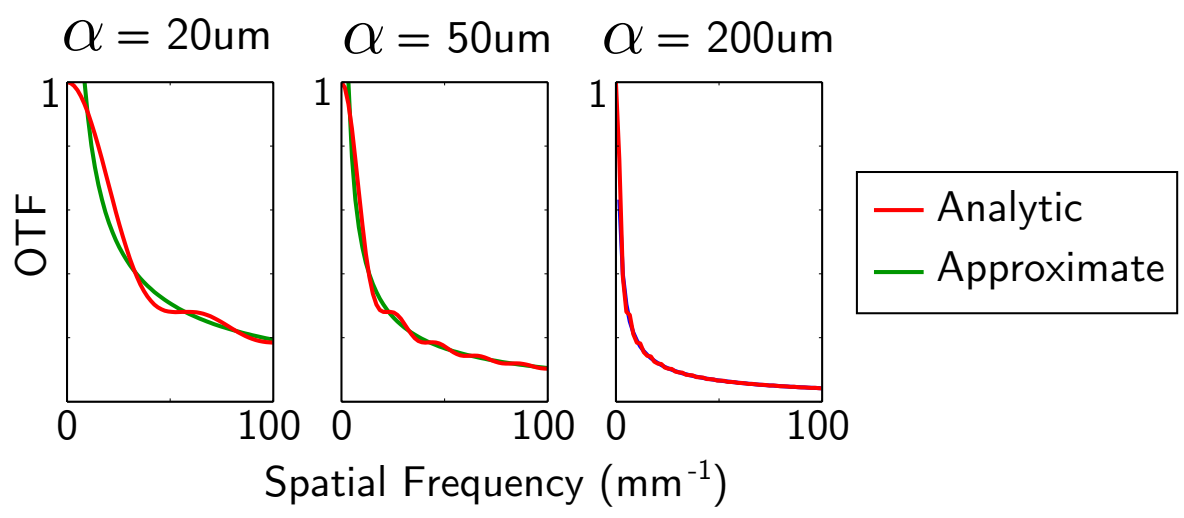

Fig. 8. A comparison of the OTF for a lens with spherical aberration calculated using using our analytic formula (red curves) and using the approximation for the OTF given by Equation 27. The OTF is calculated at various lens scales corresponding to spherical aberration coefficients of $\alpha=\{20 \mu m, 50 \mu m, 200 \mu m\}$. As the amount of spherical aberrations increase, the approximation increases in accuracy. 


\section{Deblurring Error vs. Spherical Aberration}

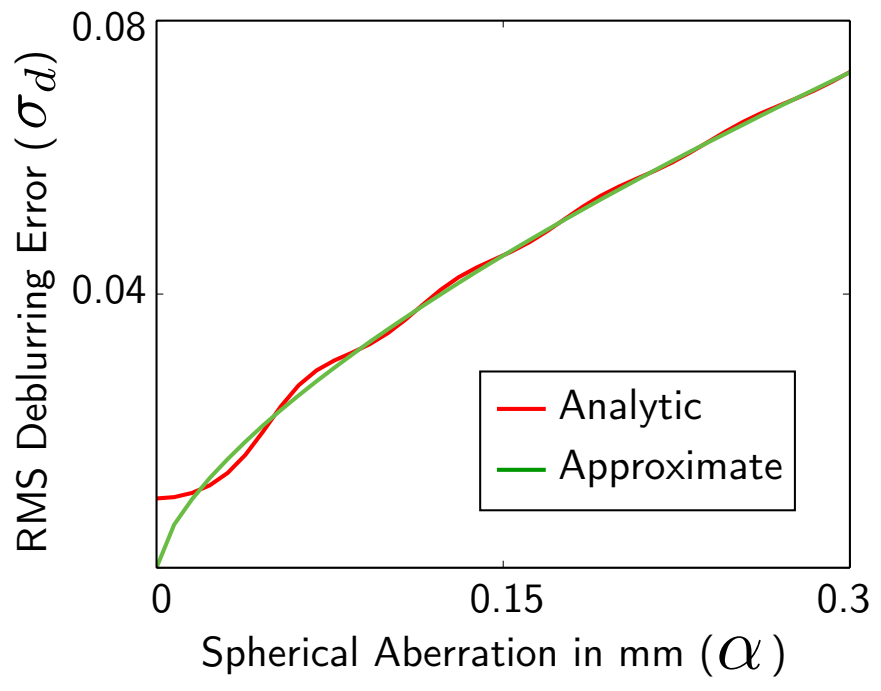

Fig. 9. A comparison of the RMS deblurring error $\sigma_{d}$ as a function of the spherical aberrations coefficient $(\alpha)$ with sensor noise $\sigma_{n}=.01$ and nyquist frequency $\Omega=100 \mathrm{~mm}^{-1}$. The red curve shows the error computed numerically using Equations 24 and 26. The green curve is calculated using the closed form expression for deblurring error given in Equation 29. The green curve closely approximates the green curve, with accuracy increasing as $\alpha$ increases.

Since we know from Equation 7 that scaling a lens by a factor of $M$ also scales $\alpha$ by the same factor, Equation 29 gives us the relation

$$
\sigma_{d}=k \sigma_{n} M^{2 / 3}
$$

where $k$ is a constant. Equation 30 expresses a remarkable fact: for lenses with spherical aberrations, while the size of the PSF increases linearly with lens scale $M$, the deblurring error increases sub-linearly. While Equation 30 is based on an approximation of the geometric OTF, it closely approximates the deblurring error calculated numerically using the OTF from Equation 24 (see Figure 9).

\section{A Scaling Law for Computational Imaging}

\section{A. Deblurring Error vs. Resolution}

For the scaling laws given in Section 5, it is assumed that the minimum resolvable spot size is equal to the blur size due to geometric aberrations, $\delta_{g}$. For a computational imaging system 
(i.e., with deblurring), the resolution is given by the pixel size $\xi$, and SBP does not depend directly on the geometric blur radius $\delta_{g}$. A more pertinent quantity for measuring image quality is SNR. In the absence of any noise we can theoretically increase SBP by decreasing pixel size until we have reached the diffraction limit. In order to provide a fair comparison between any two computational imaging systems, we must fix the SNR.

By fixing SNR, we establish a relationship between the deblurring error and pixel size. To show this, we express deblurring error as a function of lens scale $M$. Assuming the deblurring error is proportional to sensor noise, we can write

$$
\sigma_{d}=\sigma_{n} f(M)
$$

where $f(M)$ represents the scale-dependent deblurring factors. In order to force the SNR to remain constant across lens scale, we must adjust the sensor noise appropriately.

We now relate pixel size $\xi$ to sensor noise $\sigma_{n}$. Here we assume that pixels receive sufficient light such that poisson noise dominates. Then the measurement noise can be well approximated by additive gaussian noise with variance proportional to the mean signal intensity [32]. Scaling $\xi$ by a factor of $M$ increases the pixel's area by a factor of $M^{2}$. For a fully saturated pixel, assuming a shot noise limited sensor, this will increase the sensor's full well capacity by $M^{2}$ and decrease noise by a factor of $1 / M$ relative to the signal. The sensor noise is then inversely proportional to pixel size so that

$$
\xi(M) \propto \frac{1}{\sigma_{n}(M)} \propto \frac{1}{f(M)} .
$$

Equation 32 says that in order to make SNR scale independent, the pixel size should be increased as a function of $M$ to exactly cancel out scale-dependent deblurring factors. The number of resolvable points for a computational imaging systems is then

$$
R_{\text {comp }}(M)=\frac{M^{2} \Delta x \Delta y}{(\lambda F / \#)^{2}+\xi(M)^{2}} .
$$

\section{B. An Analytic Scaling Law}

Using the expression for deblurring error for a lens with spherical aberrations given by Equation 30, we see that in order to produce a SNR that is independent of lens scale, the

pixel size should be scaled according to the relation $\xi \propto M^{2 / 3}$. Plugging this into Equation 33 gives an analytic scaling law for computational imaging systems:

$$
R_{a n a}(M)=\frac{M^{2} \Delta x \Delta y}{(\lambda F / \#)^{2}+k_{2}^{2} M^{4 / 3}}
$$


where we have gathered proportionality constants in to $k_{2}$. For large $M$, the scaling law has the behavior

$$
R_{\text {ana }}(M) \propto M^{2 / 3}
$$

As with conventional lens design curve $R_{\text {conv }}$, Equation 34 gives a scaling law that breaks the resolution threshold imposed by the aberrations limit (see the magenta curve in Figure 11). However, the analytic scaling law does not behave as close to the ideal diffraction limited scaling law as the $R_{\text {conv }}$ curve. At the same time, the $R_{\text {conv }}$ curve assumes that $\mathrm{F} / \#$ reduces and more light is sacrificed as scale increases, while the $R_{a n a}$ curve does not make this assumption.

\section{C. Image Priors for Improved Performance}

In the previous section we showed analytically that, when a computational approach is taken, the resolution of a lens with spherical aberrations breaks the classical limit that results when considering geometrical spot size alone. The $R_{\text {ana }}$ curve given in Equation 34, however, does not increase as rapidly with lens scale as does Lohmann's scaling law for conventional lens designs. We now show that the scaling behavior of computational imaging systems surpasses that of conventional lens designs when image priors are taken into account.

In Section 6.A we used Equation 19 to form an estimate of our unknown image. This solution can be seen to be equivalent to the solution found by maximizing the likelihood for the probability distribution [33]

$$
P(\hat{\mathbf{y}} \mid \hat{\mathbf{x}})=\exp \|\hat{\mathbf{y}}-\Lambda \hat{\mathbf{x}}\|^{2}
$$

The maximum likelihood solution minimizes the probability of error in the estimate when no information about the the prior distribution $P(\hat{\mathbf{x}})$ is available a priori. In our case however, some information about $P(\hat{\mathbf{x}})$ is known ahead of time since the unknown quantity $\hat{\mathbf{x}}$ belongs to the class of natural images. To make a solution to the estimation problem analytically tractable, we assume a linear distribution on Fourier coefficients of natural images taking the form $P(\hat{\mathbf{x}})=\exp \|B \hat{\mathbf{x}}\|^{2}$, where $B$ is a diagonal matrix. We define the vector of Fourier coefficients $\hat{\mathbf{b}}$ such that $B_{i i}=\hat{b}_{i}$. Given a prior distribution, the maximum a posteriori solution minimizes the probability of error in the estimate. The estimate then becomes 


$$
\begin{aligned}
\hat{\mathbf{x}}^{*} & =\underset{\mathbf{x}}{\arg \max } P(\hat{\mathbf{y}} \mid \hat{\mathbf{x}}) P(\hat{\mathbf{x}}) \\
& =\underset{\mathbf{x}}{\arg \max }\left(\|\hat{\mathbf{y}}-\Lambda \hat{\mathbf{x}}\|^{2}+\|B \hat{\mathbf{x}}\|^{2}\right) \\
& =\left(\Lambda^{2}+B^{2}\right)^{-1} \Lambda^{t} \hat{\mathbf{y}},
\end{aligned}
$$

which can be written as the set of linear equations

$$
\hat{x}_{i}^{*}=\frac{\overline{\hat{p}}_{i}}{\left\|\hat{p}_{i}\right\|^{2}+\left\|\hat{b}_{i}\right\|^{2}} \hat{y}_{i},
$$

where the ${ }^{-}$operator denotes complex conjugate. We define the average power spectrum $\hat{\mathbf{a}}$ such that $\hat{a}_{i}=E\left[\left\|\hat{x}_{i}\right\|^{2}\right]$, where the expectation is taken with respect to the set of natural images. Then, as Zhou and Nayar showed, the optimal vector $\hat{\mathbf{b}}$ is such that $\hat{b}_{i}=\sigma_{n}^{2} / \hat{a}_{i}$, and the squared deblurring error becomes [34]

$$
\sigma_{d}^{2}=\sigma_{n}^{2} \sum_{i=1}^{N} \frac{1}{\left\|\hat{p}_{i}\right\|^{2}+\sigma_{n}^{2} / \hat{a}_{i}} .
$$

Figure 10 shows the deblurring error $\sigma_{d}$ calculated using Equations 24 and $41 . \sigma_{d}$ is shown as a function of spherical aberration $\alpha$ for a variety of sensor noise levels in the range $\sigma_{n}=[.002, .1]$. A polynomial is fit to each curve, and the best fit is found to be in the range $\sigma_{d} \propto \alpha^{1 / 3.4}$ to $\sigma_{d} \propto \alpha^{1 / 4.2}$. We approximate the deblurring error as

$$
\begin{aligned}
\sigma_{d} & \propto \sigma_{n} \alpha^{1 / 3.8} . \\
& \propto \sigma_{n} M^{1 / 3.8} .
\end{aligned}
$$

In fact, this estimate is slightly pessimistic, as the deblurring error also increases sub-linearly with $\sigma_{n}$ as well as $\alpha$. From Equations 43 and 33, we conclude that when image priors are used for deblurring, the resolution of a computational imaging system obeys the scaling law given by (see the cyan curve in Figure 11)

$$
R_{\text {prior }}(M)=\frac{M^{2} \Delta x \Delta y}{(\lambda F / \#)^{2}+k_{3}^{2} M^{2 / 3.8}}
$$

where again we have gathered proportionality constants into $k_{3}$. While the analytic scaling law curve $R_{\text {ana }}$ does not scale as quickly as the conventional lens design curve $R_{\text {conv }}$, the 


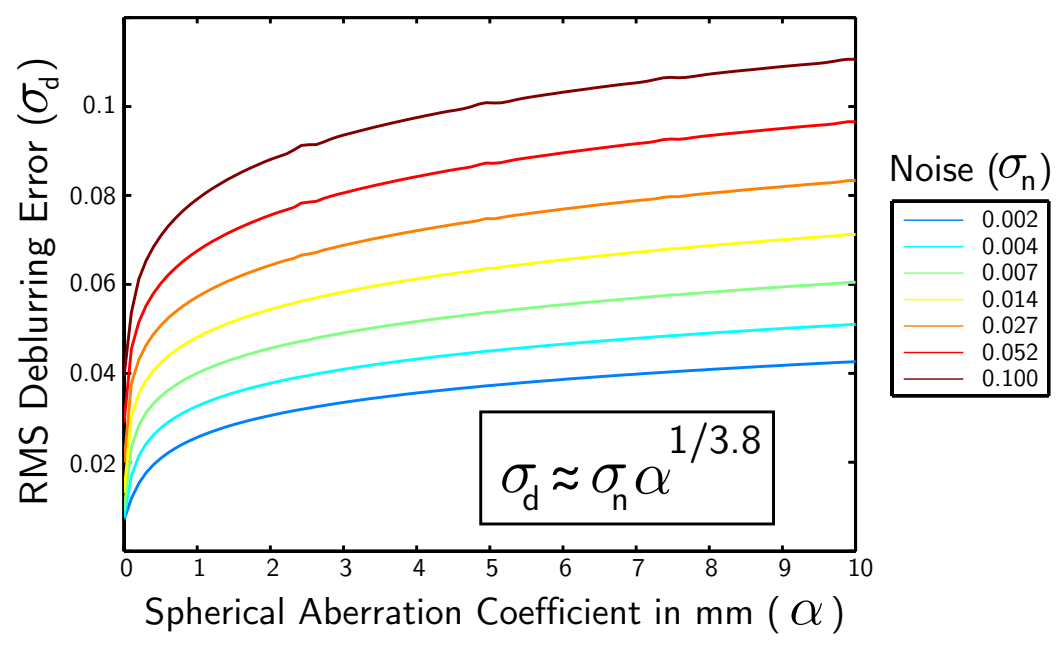

Fig. 10. RMS deblurring error as a function of spherical aberration $(\alpha)$. As $\alpha$ increases, both the PSF size and the deblurring error increase. While the size of the PSF increases linearly with $\alpha$, deblurring error increases with $\alpha^{1 / 3.8}$. In this experiment, the nyquist frequency $\Omega=250 \mathrm{~mm}^{-1}$.

curve $R_{\text {prior }}$ scales more quickly. From this we conclude that in building a camera at a desired resolution, when image priors are taken into account, a computational camera can be built at a smaller scale than a conventional lens design. Again, the $R_{\text {conv }}$ curve assumes that $\mathrm{F} / \#$ reduces and more light is sacrificed as scale increases, while the $R_{\text {prior }}$ curve does not make this assumption.

\section{Gigapixel Computational Cameras}

According to Equation 44, a computational imaging approach can enable a greater resolution to be achieved with a smaller camera size. To demonstrate this principle, we show results from a proof of concept camera that utilize a very simple optical element. By using a large ball lens, an array of planar sensors, and deconvolution as a post processing step, we are able to capture gigapixel images with a very compact camera.

The key to our architecture lies in the size of the sensors relative to the ball lens. Together, a ball lens and spherical image plane produce a camera with perfect radial symmetry. We approximate a spherical image plane with a tessellated regular polyhedron, such as an icosahedron. A planar sensor is placed on each surface of the polyhedron. Note that because sensors are typically rectangular, a different polyhedron, such as a truncated icosahedron, may provide more optimal sensor packing. Relatively small sensors are used so that each sensor occupies a small FOV and the image plane closely approximates the spherical surface. 


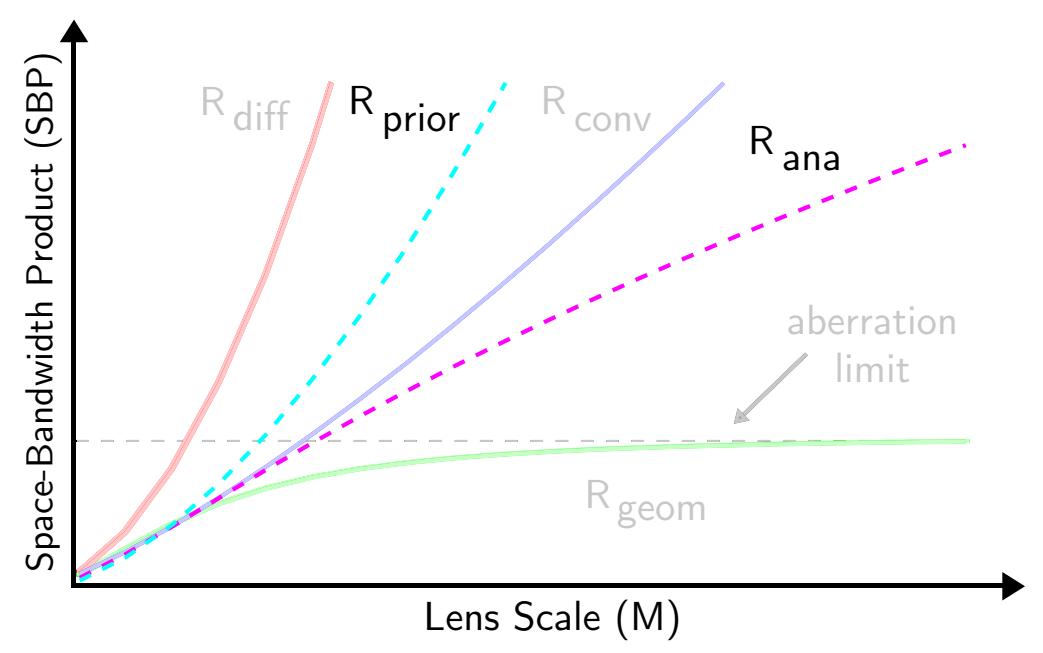

Fig. 11. Scaling laws for computational imaging systems with spherical aberrations. The $R_{\text {ana }}$, which was analytically derived, shows an improvement upon the aberration limited curve $R_{\text {geom }}$, without requiring F/\# to increase with $M$. Performance is further improved when natural image priors are taken into account, as the $R_{\text {prior }}$ curve shows. The $R_{\text {prior }}$ curve improves upon the conventional lens design curve $R_{c o n v}$, also without requiring $\mathrm{F}$ /\# to increase with $M$.

As a result, our camera produces a PSF that is not completely spatially invariant, but comes within a close approximation.

\section{A. A Proof-of-Concept Gigapixel Camera}

The first system we demonstrate consists solely of a ball lens and an array of planar sensors. We use a $100 \mathrm{~mm}$ acrylic ball lens and a 5 megapixel 1/2.5" Lu575 sensor from Lumenera [35] (see Figure 12(a)). We emulate an image captured by multiple sensors by sequentially scanning the image plane using a pan/tilt motor. With this camera, a 1 gigapixel image can be generated over a roughly $60^{\circ} \times 40^{\circ}$ FOV by tiling $14 \times 14$ sensors onto a $75 \mathrm{mmx} 50 \mathrm{~mm}$ image surface. When acquiring images with the pan/tilt unit, we allow a small overlap between adjacent images.

The PSF as a function of field position on each individual sensor is shown in Figure 12(b). Note that the PSF shape remains fairly consistent across the FOV of each sensor. The MTF (shown in in Figure 12(c)) avoids zero crossings up to the Nyquist frequency of the sensor. The plots were generated using Zemax Optical Design Software [31].

An implementation of this design is shown in Figure 13. Figures 2, 14, and 16 show 


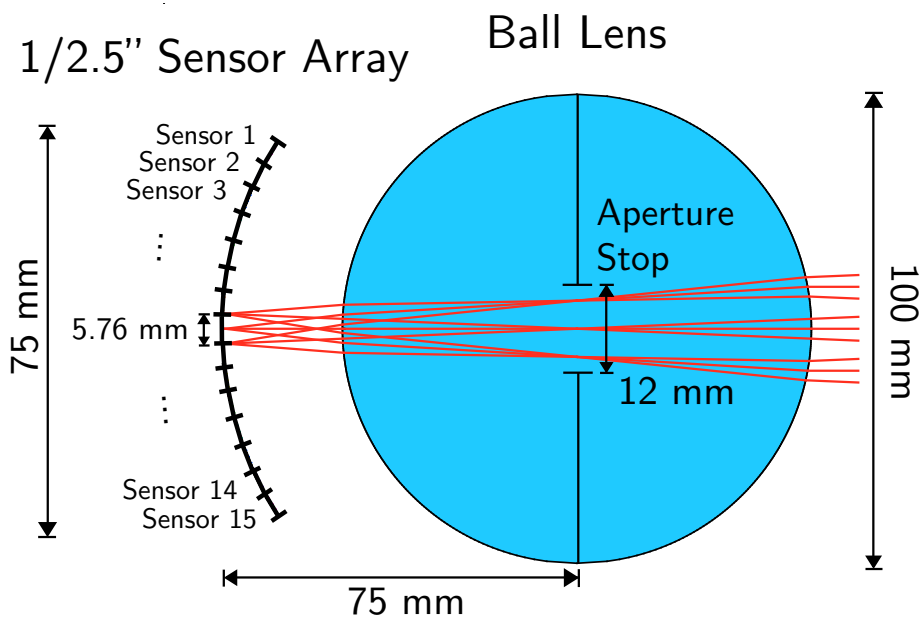

(a) An F/4 75mm focal length ball lens system.

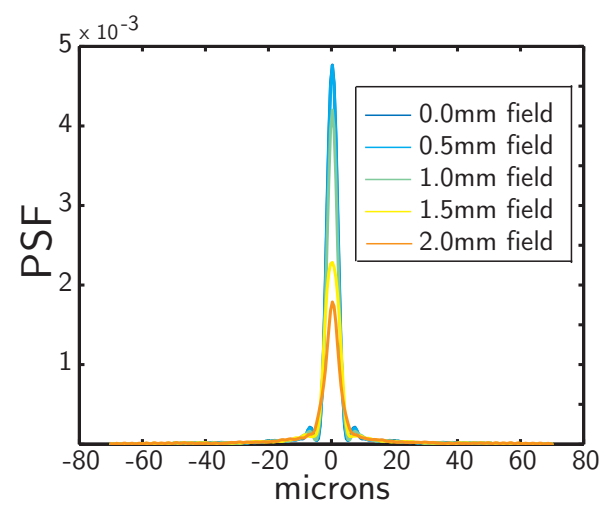

(b) The system PSF of (a)

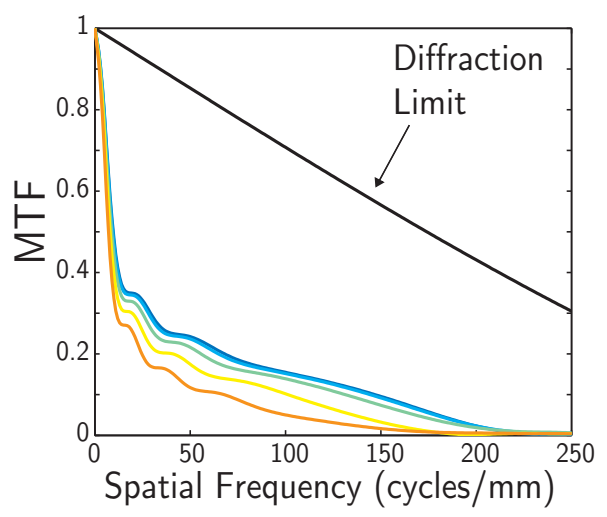

(c) The system MTF of (a)

Fig. 12. (a) Our single element gigapixel camera, which consists solely of a ball lens with an aperture stop surrounded by an array of planar sensors. (b) Because each sensor occupies a small FOV, the PSF is nearly invariant to field position on the sensor. (c) The PSF is easily invertible because the MTF avoids zero crossings and preserves high frequencies.

two gigapixel images captured with this system. Note the remarkable level of detail captured in each of the photographs. Zooming in to Figure 2 reveals the label of a resistor on a PCB board, the stippling print pattern on a dollar bill, a miniature 2D barcode pattern, and the extremely fine ridges of a fingerprint. Closeups in Figure 14 reveal fine details in a watch, an eye, a resolution chart, and individual strands of hair. Closeups in Figure 16 reveal details that are completely invisible in the zoomed out panorama, including a sailboat, a sign 


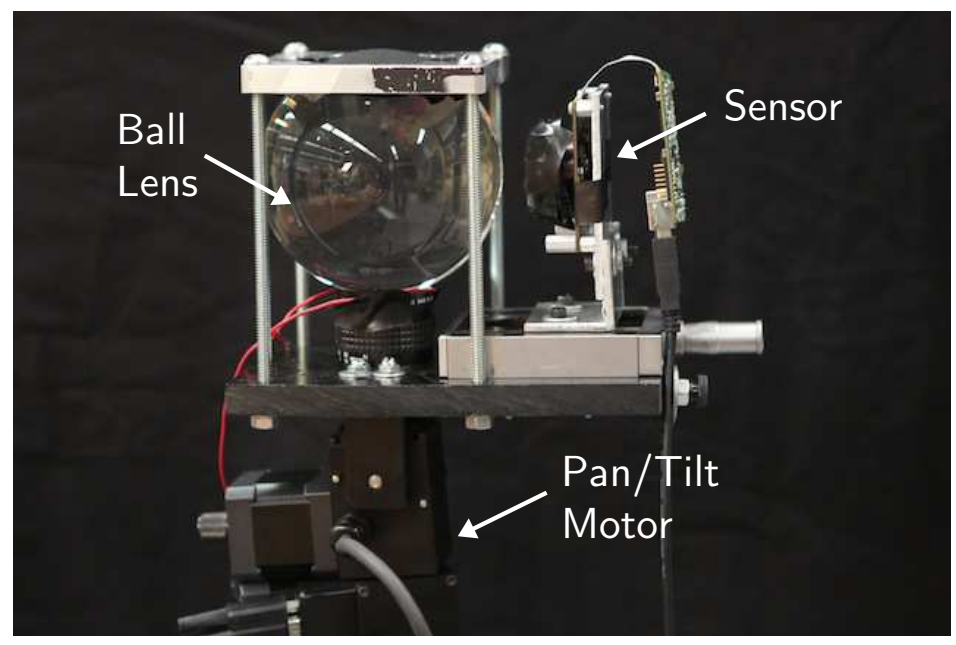

Fig. 13. A system used to verify the performance of the design shown in Figure 12(a). An aperture is placed on the surface of the ball lens. A gigapixel image is captured by sequentially translating a single 1/2.5", 5 megapixel sensor with a pan/tilt motor. A final implementation would require a large array of sensors with no dead space in between them.

advertising apartments for sale, the Empire State Building, and cars and trucks driving on a bridge

\section{A.1. Color}

Because our cameras do not include any color correcting elements, they suffer from axial chromatic aberrations. For our $100 \mathrm{~mm}$ diameter ball lens that we use, the chromatic focus shift is about $1.5 \mathrm{~mm}$ over the visible wavelength range. However, most of the image blur caused by chromatic focus shift is in the chrominance channel of captured images [6] [7]. Since humans are less sensitive to blur in chrominance channels, axial chromatic aberrations do not cause a significant degradation in perceived image quality. We use the deblurring technique from Cossairt and Nayar [7], which is inexact but produces images that look good.

\section{A.2. $\quad$ Post Processing}

The post processing for captured images follows several steps. First, a transformation from RGB to YUV color space is applied. Next, Wiener deconvolution is applied to the luminance channel only, and the image is transformed back to RGB color space. A noise reduction algorithm is then applied to suppress deblurring artifacts. We found the BM3D algorithm [36] to produce the best results. Finally, the set of captured images are stitched to obtain a high 


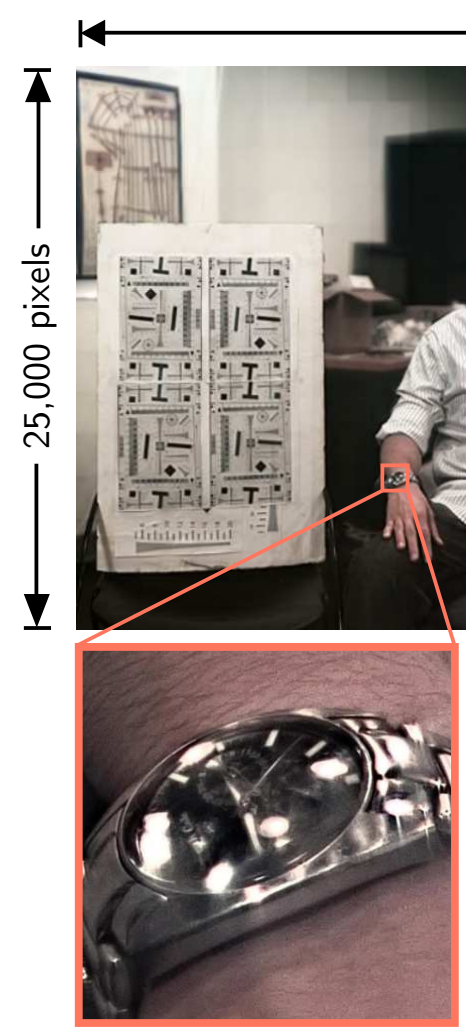

Watch

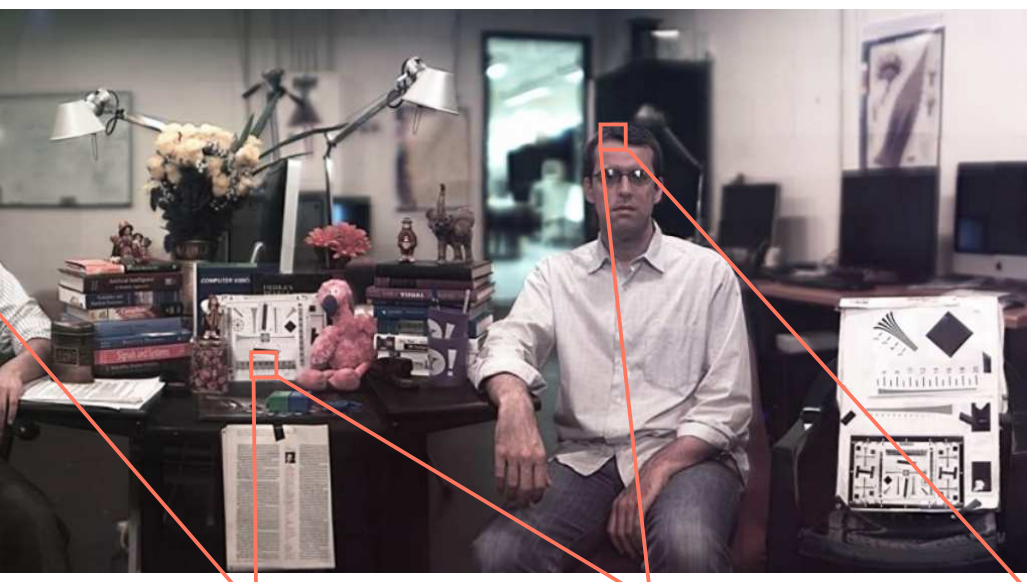

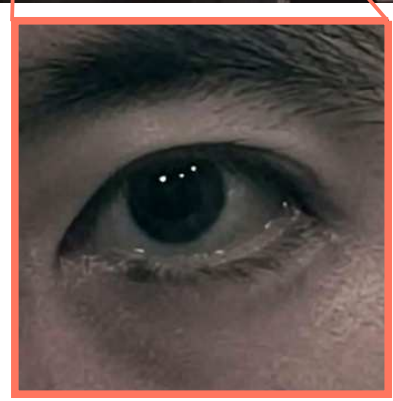

Eye

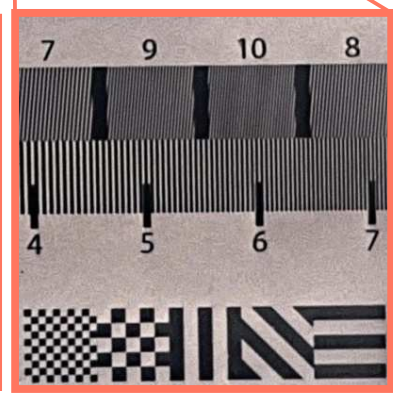

Resolution Chart

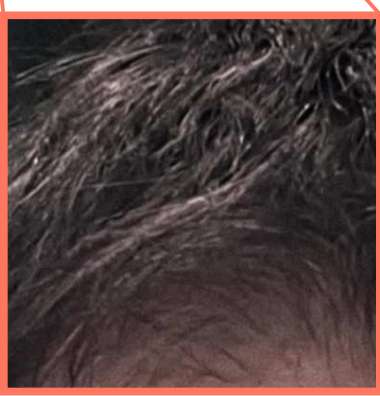

Hair

Fig. 14. A 1.6 gigapixel image captured using the implementation shown in Figure 13. The image dimensions are 65,000 x 25,000 pixels, and the scene occupies a $104^{\circ} \times 40^{\circ} \mathrm{FOV}$. From left to right, the insets reveal fine details in a watch, an eye, a resolution chart, and individual strands of hair. Please visit http://gigapan.org/gigapans/09d557515ee4cc1c8c2e33bf4f27485a/ to view this example in more detail.

resolution image using the Microsoft Image Composite Editor [37].

\section{B. A Single Element Design}

The design in Figure 12(a) is extremely compact, but impractical because adjacent sensors must be packed without any dead space in between them. The size of this system is limited by the package size of the sensor relative to the active sensor area. Sensors with a package size that is only $1.5 x$ larger than the active sensor area are currently commercially available. With these sensors, it is possible to build a gigapixel camera that uses only a single optical element, as shown in Figure 15(a). In this design, each sensor is coupled with a smaller acrylic relay lens that decreases the focal length of the larger acrylic ball lens. The relay 
lenses share a surface with the ball lens, which means that it is possible to combine the entire optical system into a single element that may be manufactured by molding a single material, drastically simplifying the complexity (and hence alignment) of the system.

\section{C. Capturing the Complete Sphere}

All the designs proposed in this paper use a ball lens. A great advantage of using a ball lens is that, because it has perfect radial symmetry, a near hemispherical FOV can be captured. In fact, it can even be used to capture the complete sphere, as shown in Figure 15(b). This design is similar to the one in Figure 15(a) with a large gap between adjacent lens/sensor pairs. Light passes through the gaps on one hemisphere, forming an image on a sensor located on the opposite hemisphere. As a result, the sensors cover the complete $2 \pi$ FOV at the cost of losing roughly half the incident light.

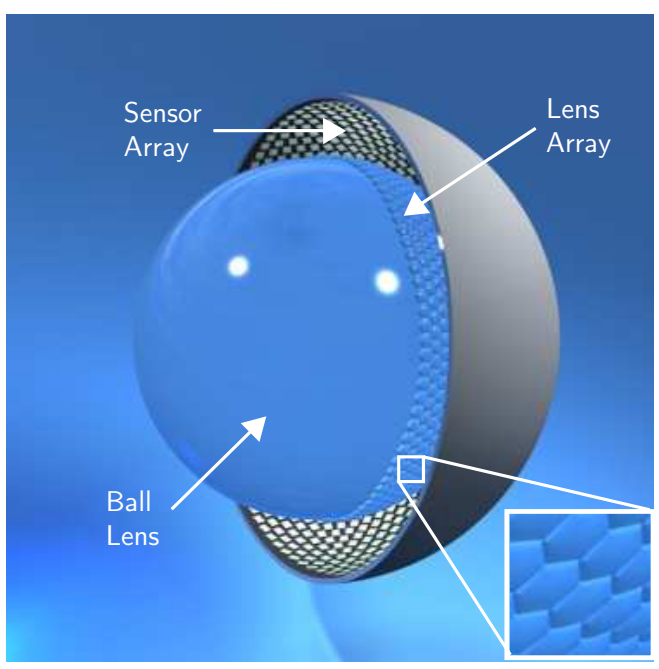

(a) A single element design

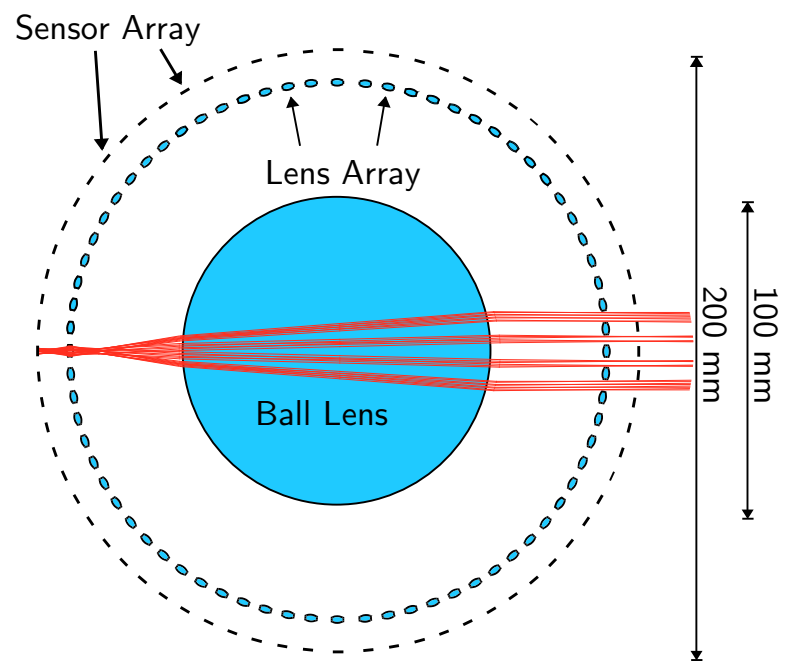

(b) A $4 \pi$ FOV design

Fig. 15. (a) A single element design for a gigapixel camera. Each sensor is coupled with a lens that decreases focal distance, allowing FOV to overlap between adjacent sensors. (b) A design for a gigapixel camera with a $2 \pi$ radian FOV. The design is similar to the implementation in Figure 15(a) with a large gap between adjacent lens/sensor pairs. Light passes through the gaps on one hemisphere, forming an image on a sensor located on the opposite hemisphere. 


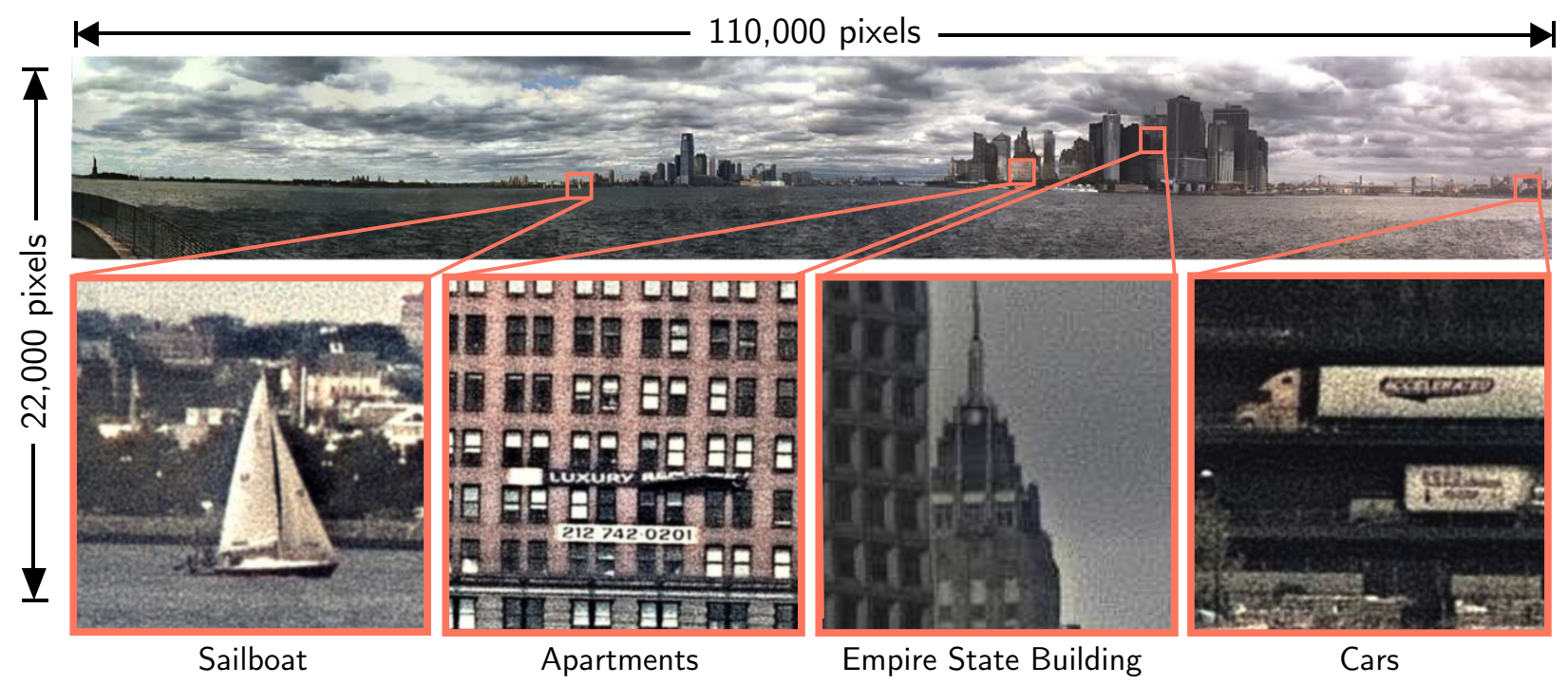

Fig. 16. A 1.4 gigapixel image captured using the implementation shown in Figure 13. The image dimensions are $110,000 \times 22,000$ pixels, and the scene occupies a $170^{\circ} \times 20^{\circ}$ FOV. From left to right, insets reveal a sailboat, a sign advertising apartments for sale, the Empire State Building, and cars and trucks driving on a bridge. Please visit http://gigapan.org/gigapans/7173ad0acace87100a3ca728d40a3772/ to view this example in more detail.

\section{Discussion}

\section{A. Limitations of Scaling Laws}

In this paper, scaling laws were derived which express the the general scaling behavior of resolution versus lens scale $M$, with special attention paid to how the behavior for increasingly large values of $M$. However, because we have chosen to speak in general terms about the scaling behavior, we have not given attention to how resolution behaves for smaller values of $M$, which may result in different behavior. For instance, when $M$ is large, conventional lens designs outperform computational imaging without priors, as indicated by the $R_{\text {conv }}$ and $R_{\text {ana }}$ curves. However, for small $M, R_{\text {ana }}$ may actually be greater than $R_{\text {conv }}$, depending on the exact values of the proportionality constant $k_{1}$ and the amount of spherical aberration $\delta_{g}$. These exact values will vary depending on the specific lens design and sensor characteristics, but the aggregate behavior for large values of $M$ will will remain consistent accross all scenarios. In this way, the scaling laws encompass the gross behavior of lenses and sensors, but do not always lend themselves to a direct comparison between specific designs. 


\section{B. On Computational Imaging and Scaling Laws}

The original scaling laws derived by Lohmann are pleasingly simple in the sense that they keep the problem domain constrained to a single variable: the scale parameter $M$. In some sense, introducing computational imaging made the problem more complicated because it introduced a new variable in the form of SNR. Looking at the problem in a general way, the resolution scaling behavior of different imaging systems can vary both as a function of lens scale and SNR. While Lohmann made no mention of SNR in his original analysis, there was an implicit relationship between SNR and resolution that was unstated. For example, consider the expression for the scaling behavior of lenses in the presence of geometric aberrations given by Equation 13. We recall that, for large $M$, resolution plateaus at $\Delta x \Delta y / \delta_{g}$. However, if we choose to match pixel area to blur area, then pixel size increases linearly with $M$. Thus, according to the arguments in Section 7 , if we continue to scale a lens beyond the aberration limit, resolution does not increase, while SNR increases linearly with $M$. On the other hand, for diffraction limited lenses, pixel size, and thus SNR, remains constant while resolution scales quadratically with lens scale. This leads to an interesting observation about the tradeoff between resolution and SNR. In some sense, these two examples are opposite extremes in a two-dimensional design space. When geometric aberrations are present, resolution becomes fixed but SNR can increase, while for diffraction limited lenses, SNR becomes fixed but resolution can increase.

This brings us to the scaling laws for conventional lens design and computational imaging. The conventional lens design curve, $R_{\text {conv }}$, is derived assuming that both $\mathrm{F} / \#$ and pixel size increase with $M^{1 / 3}$. In the photon limited noise regime, SNR is proportional to pixel size $\xi$, and inversely proportional to $\mathrm{F} / \#$. Thus, while the $R_{c o n v}$ curve is derived assuming that more light is sacrificed as lens scale increases, the amount of photons collected per pixel remains fixed, and thus so does SNR. Similarly, in the computational imaging regime, we ask what pixel scaling behavior will produce a deblurring error, and hence SNR, that is independent of lens scale.

The scaling laws for computational imaging and conventional lens design represent the behavior of two competing techniques that are trying to achieve the same goal: maximizing resolution scaling behavior while fixing SNR. Neither technique achieves the ideal scaling performance of diffraction limited lenses. In effect, both techniques are complexity reducing measures, since they aim to maximize performance without introducing the added optical elements required to reduce aberrations below the diffraction limit. This brings us to a third axis in our design space: lens complexity. As we scale a diffraction limited lens, SNR remains fixed and resolution reaches the maximum scaling potential, however lens complexity must also increase in an effort to combat greater amounts of geometrical aberrations. In contrast, for the computational imaging and conventional lens scaling laws, both SNR and 

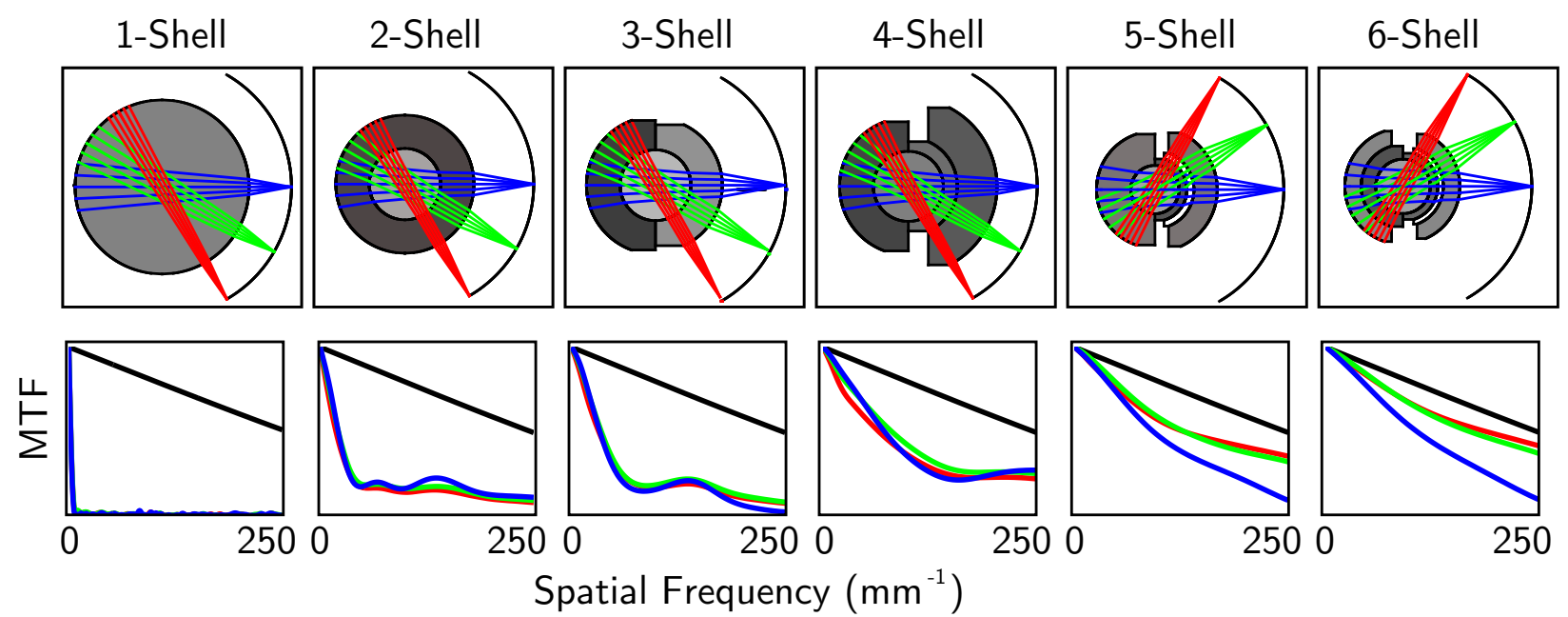

Spatial Frequency $\left(\mathrm{mm}^{-1}\right)$

Fig. 17. The MTF for spherical optical systems with varying amounts of complexity. Complexity is measured as the number of optical surfaces, which increases from left to right as 1 to 6 surfaces. The six surface design is the Gigagon lens designed by Marks and Brady [21]. Each design is a F/2.8 280mm FL lens optimized using Zemax. As the number of surfaces increases, the MTF improves, improving the SNR as well.

lens complexity remain fixed, but the maximum scaling potential is not achieved.

In an ideal setting, we would like to maximize resolution and SNR while minimizing lens scale and complexity. This cannot be achieved in practice, however, and the best that can be done is to develop a merit function that weighs these measures in terms of their relative importance on an application dependent basis. Lens optimization based on this merit function then gives the design which results in the best performance for this specific application.

\section{C. The Performance vs. Complexity Trade-off}

According to Equation 44, with the aid of computations, the resolution of a lens with spherical aberrations will, in general, scale more quickly than for a conventional lens design. However, a lens which requires deblurring will have a smaller SNR than a diffraction limited lens of the same scale. For the designs proposed in Section 8, we have chosen designs that favor simplicity, and as a consequence, also result in a lower SNR. Any computational imaging system poses an inherent trade-off between complexity and SNR. In practice, exploring this trade-off requires a carefully designed measure for complexity.

A good complexity measure must take into account many different factors: the number of 
surfaces, the degree polynomial of each surface, etc. While it is difficult to develop a general measure for complexity that applies to all lens designs, the problem becomes much simpler when we consider only concentric spherical optical elements. In this case, complexity can simply be quantified as the number of surfaces used in the design.

To explore the tradeoff between complexity and SNR for the special case of spherical optics, we created six spherical optics designs, ranging in complexity from 1 shell to 6 shells. The six designs were created in an effort to analyze how the best case performance of a computational imaging system scales as a function of lens complexity. Shells 1-5 were optimized with Zemax using a custom optimization procedure that minimizes the deblurring error. The six shell design shown is the Gigagon lens designed by Marks and Brady [21]. The six designs and their relative performance are shown in Figure 17. From the MTF plots shown at the bottom of the figure, it can be seen that the six shell design performs near diffraction limited, and the MTF steadily decreases with decreasing complexity.

Figure 18 shows how, rather than favoring simplicity, an optimal design may consist of more

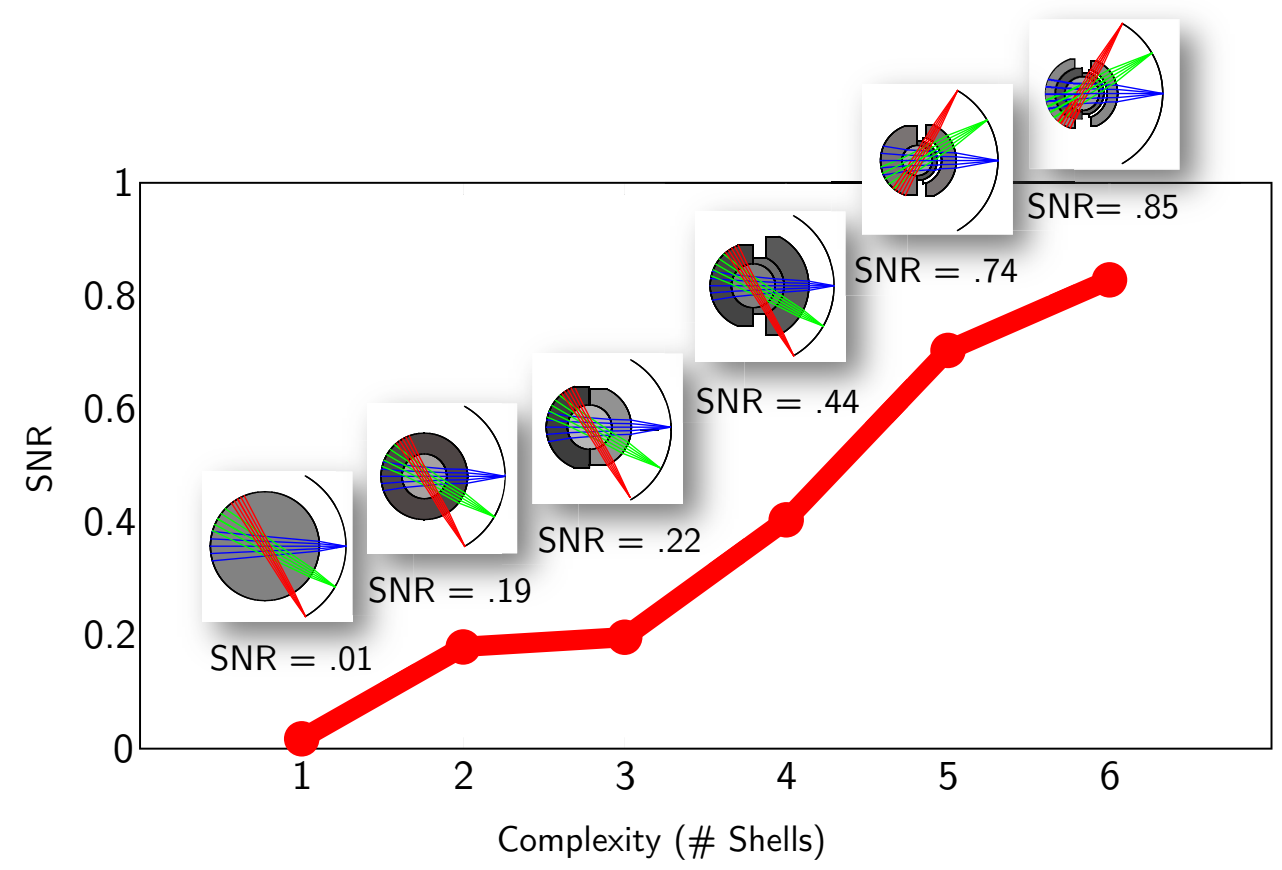

Fig. 18. SNR vs. complexity for the lens designs shown in Figure 18, assuming a computational approach is taken. SNR increases by a factor of 19 when complexity increases from 1 shell to 2 shells, while SNR only increases by a factor of 4 when complexity increases from 2 shells to 6 shells. 
elements than the designs discussed previously in this paper. It appears that, for the special case of spherical optics, there is a law of diminishing returns when it comes to improving performance by means of increasing complexity. In particular, we note that SNR increases by a factor of 19 when complexity increases from 1 shell to 2 shells, while SNR only increases by a factor of 4 when complexity increases from 2 shells to 6 shells. Taking this behavior in to account, an optimal design may be found by balancing lens scale and complexity. Such a design would have minimum scale and complexity for a given resolution, and a fixed scale/complexity ratio. Whether or not general lens designs also have similar complexity vs. performance behavior is an open question that is currently under investigation.

\section{Conclusion}

We have given a comprehensive analysis on the resolution scaling behavior of cameras with respect to lens size. Lohmann's original results have been extended to include the resolution scaling behavior of computational imaging systems, with special attention paid to lenses that exhibit spherical aberrations. Closed form expressions for the PSF, OTF, and deblurring error of lenses which exhibit spherical aberrations have been derived. In addition, we have shown that, when image priors are taken into consideration, computational imaging systems exhibit superior scaling performance with respect to conventional lens designs. The result gives credence to the further development of computational imaging techniques developed for the purpose of reducing lens complexity.

In support of our analysis on resolution scaling behavior, we have explored the design of gigapixel computational imaging systems based on spherical optical elements, which primar-

ily exhibit spherical aberrations. We demonstrated a proof-of-concept system that emulates a spherical lens surrounded by an array of planar sensors tessellated onto a spherical surface. Several examples were shown which demonstrate promising image quality with a compact camera that exhibits low lens complexity. Finally, the trade-off between performance and lens complexity is explored, providing a complete exploration of the design space of resolution, SNR, lens scale and complexity for the special case of spherical optics.

\section{Appendix A: PSF Derivation}

From Equations 8 and 11, the PSF produced by a lens with a monomial OPD is given by

$$
P(r)=\int_{-\infty}^{\infty} \sqcap(\rho) \frac{\delta\left(r-\alpha \rho^{n}\right)}{\pi|r|}|\rho| d \rho
$$

We introduce a change of variables $z=\alpha \rho^{n}$, giving the relations 


$$
\begin{aligned}
\rho & =\left(\frac{|z|}{\alpha}\right)^{1 / n} \\
d \rho & =\frac{1}{n \alpha}\left(\frac{|z|}{\alpha}\right)^{1 / n-1} d z .
\end{aligned}
$$

After substitution, the PSF becomes

$$
\begin{aligned}
P(r) & =\frac{1}{\pi|r|} \int_{-\infty}^{\infty} \sqcap\left(\frac{z}{\alpha}\right) \delta(r-z) \frac{1}{n \alpha}\left(\frac{|z|}{\alpha}\right)^{1 / n}\left(\frac{|z|}{\alpha}\right)^{1 / n-1} d z \\
& =\frac{1}{\pi|r| n \alpha} \int_{-\infty}^{\infty} \sqcap\left(\frac{z}{\alpha}\right) \delta(r-z)\left(\frac{|z|}{\alpha}\right)^{2 / n-1} d z \\
& =\frac{1}{\pi|r| n \alpha} \sqcap\left(\frac{r}{\alpha}\right)\left(\frac{|r|}{\alpha}\right)^{2 / n-1} \\
& =\frac{1}{\pi n \alpha^{2 / n}} \sqcap\left(\frac{r}{\alpha}\right)|r|^{2 / n-2} .
\end{aligned}
$$

\section{Appendix B: PSF Normalization}

The energy for the PSF given in Equation 11 is

$$
\begin{aligned}
e & =\pi \int_{-\infty}^{\infty} P_{r}(r)|r| d r \\
& =\pi \int_{-\infty}^{\infty} \frac{1}{\pi n \alpha^{2 / n}} \sqcap\left(\frac{r}{\alpha}\right)|r|^{2 / n-2}|r| d r \\
& =\frac{1}{n \alpha^{2 / n}} \int_{-\alpha}^{\alpha}|r|^{2 / n-1} d r \\
& =\frac{1}{n \alpha^{2 / n}}\left[\frac{n}{2} \frac{r}{|r|}|r|^{2 / n}\right]_{\alpha}^{-\alpha} \\
& =\frac{1}{n \alpha^{2 / n}}\left(n \alpha^{2 / n}\right)=1
\end{aligned}
$$

which verifies that the PSF is properly normalized,

\section{Acknowledgements}

This research was supported in part by DARPA Award No. W911NF-10-1-0214. Oliver Cossairt was supported by an NSF Graduate Research Fellowship. The authors thank Ravi Athale of Mitre Corporation for his comments, Keith Yeager of Columbia University for his help with machining, Lauren Kushner for her 3D modeling expertise, and Tai-Hsu Lin for programming assistance. 


\section{References}

1. "Darpa at 50," www.darpa.mil/Docs/1-25013846_Eprint_200811141152151.eps (2010).

2. K. Fife, A. El Gamal, and H. Wong, "A 3MPixel Multi-Aperture Image Sensor with 0.7 um Pixels in 0.11 um CMOS," in "IEEE ISSCC Conference," (2008).

3. J. Goodman, Introduction to Fourier optics (Roberts \& Company Publishers, 2005).

4. M. Robinson, G. Feng, and D. Stork, "Spherical coded imagers," in "Proc. SPIE,", vol. 7429 (2009), vol. 7429, p. 20.

5. M. Robinson and V. Bhakta, "Experimental validation of extended depth-of-field imaging via spherical coding," in "Computational Optical Sensing and Imaging," (OSA, 2009).

6. F. Guichard, H.-P. Nguyen, R. Tessières, M. Pyanet, I. Tarchouna, and F. Cao, "Extended depth-of-field using sharpness transport across color channels," (Proc. SPIE, 2009), vol. 7250.

7. O. Cossairt and S. Nayar, "Spectral focal sweep: Extended depth of field from chromatic aberrations," in "ICCP," (2010).

8. M. Ben-Ezra, "High Resolution Large Format Tile-Scan - Camera Design, Calibration, and Extended Depth of Field," in "ICCP," (2010).

9. S. Wang and W. Heidrich, "The design of an inexpensive very high resolution scan camera system," in "Computer Graphics Forum," , vol. 23 (Citeseer, 2004), vol. 23, pp. $441-450$.

10. "The Gigapixl Project," http://www.gigapixl.org/ (2007).

11. B. Wilburn, N. Joshi, V. Vaish, E. Talvala, E. Antunez, A. Barth, A. Adams, M. Horowitz, and M. Levoy, "High performance imaging using large camera arrays," ACM Transactions on Graphics (TOG) 24, 776 (2005).

12. Y. Nomura, L. Zhang, and S. Nayar, "Scene collages and flexible camera arrays," in "Proc. EGSR," (2007).

13. D. J. Brady and N. Hagen, "Multiscale lens design," Opt. Express 17, 10659-10674 (2009).

14. R. Kingslake, A history of the photographic lens (Academic Press, 1989).

15. R. Luneburg, Mathematical theory of optics (University of California Press, 1964).

16. S. Rim, P. Catrysse, R. Dinyari, K. Huang, and P. Peumans, "The optical advantages of curved focal plane arrays," in "Proc. SPIE," , vol. 5678 (2005), vol. 5678, pp. 48-58.

17. G. Krishnan and S. Nayar, "Towards A True Spherical Camera," in "SPIE Human Vision and Electronic Imaging," (2009).

18. R. Dinyari, S. Rim, K. Huang, P. Catrysse, and P. Peumans, "Curving monolithic silicon for nonplanar focal plane array applications," Applied Physics Letters 92, 091114 (2008).

19. H. Ko, M. Stoykovich, J. Song, V. Malyarchuk, W. Choi, C. Yu, J. Geddes Iii, J. Xiao, 
S. Wang, Y. Huang et al., "A hemispherical electronic eye camera based on compressible silicon optoelectronics," Nature 454, 748-753 (2008).

20. L. Lee and R. Szema, "Inspirations from biological optics for advanced photonic systems," Science 310, 1148 (2005).

21. D. Marks and D. Brady, "Gigagon: A Monocentric Lens Design Imaging 40 Gigapixels," in "Imaging Systems," (OSA, 2010).

22. E. Dowski and J. Cathey, "Extended depth of field through wave-front coding," Appl. Opt 34, 1859-1866 (1995).

23. E. Dowski Jr, R. Cormack, and S. Sarama, "Wavefront coding: jointly optimized optical and digital imaging systems," in "Proc. SPIE," , vol. 4041 (Citeseer, 2000), vol. 4041, pp. 114-120.

24. M. Robinson and D. G. Stork, "Extending depth-of-field: Spherical coding versus asymmetric wavefront coding," in "Computational Optical Sensing and Imaging," (OSA, 2009).

25. A. W. Lohmann, "Scaling laws for lens systems," Appl. Opt. 28, 4996-4998 (1989).

26. J. Geary, Introduction to lens design: with practical ZEMAX examples (Willmann-Bell, 2002).

27. A. Levin, W. Freeman, and F. Durand, "Understanding camera trade-offs through a bayesian analysis of light field projections," Computer Vision-ECCV 2008 pp. 88-101 (2008).

28. A. Levin, S. Hasinoff, P. Green, F. Durand, and W. Freeman, " 4 d frequency analysis of computational cameras for depth of field extension," in "ACM SIGGRAPH 2009 papers," (ACM, 2009), pp. 1-14.

29. O. Cossairt, C. Zhou, and S. Nayar, "Diffusion coded photography for extended depth of field," in "ACM SIGGRAPH 2010 papers," (ACM, 2010), pp. 1-10.

30. L. J. Slater, Generalized Hypergeometric Functions (Cambridge University Press, 1966).

31. "Zemax Optical Design Software," http://www.zemax.com/ (2010).

32. A. Chakrabarti, K. Hirakawa, and T. Zickler, "Computational color constancy with spatial correlations," Harvard Technical Report TR-09-10 (2010).

33. M. Bertero and P. Boccacci, Introduction to inverse problems in imaging (Taylor \& Francis, 1998).

34. C. Zhou and S. Nayar, "What are good apertures for defocus deblurring?" in "ICCP," (IEEE, 2010), pp. 1-8.

35. "Lumenera Corporation," http://www.lumenera.com/ (2010).

36. K. Dabov, A. Foi, V. Katkovnik, and K. Egiazarian, "Image denoising with blockmatching and 3 D filtering," in "Proc. SPIE," , vol. 6064 (Citeseer, 2006), vol. 6064, pp. 354-365. 
37. "Microsoft Image Composite Editor (ICE) website," http://research.microsoft.com/enus/um/redmond/groups/ivm/ICE/ (2010). 